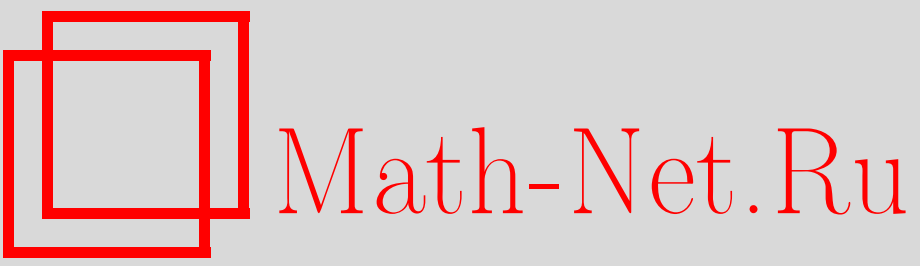

Ю. И. Сапронов, Конечномерные редукции в гладких экстремальных задачах, УМН, 1996, том 51, выпуск 1, 101-132

DOI: https://doi.org/10.4213/rm921

Использование Общероссийского математического портала Math-Net.Ru подразумевает, что вы прочитали и согласны с пользовательским соглашением

http://www . mathnet.ru/rus/agreement

Параметры загрузки:

IP: 3.91 .87 .62

26 апреля 2023 г., 13:56:04 


\section{КОНЕЧНОМЕРНЫЕ РЕДУКЦИИ В ГЛАДКИХ ЭКСТРЕМАЛЬНЫХ ЗАДАЧАХ}

Ю.И. САПРОНОВ

СОДЕРЖАНИЕ

Введение

Глава 1. Понижение размерности в конечномерных экстремальных за-

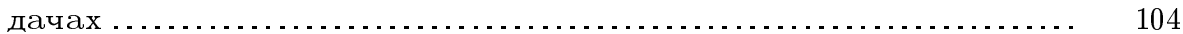

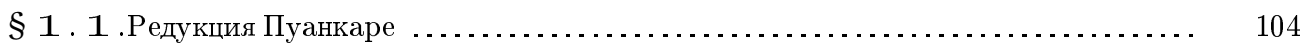

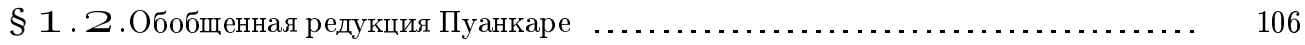

$\S 1.3$. Посткритические равновесия упругой цепи . . . . . . . . . . . . . . . . . . 109

$\S 1.4$. . тационарные вращения многомерных волчков. Редукция Смейла . . . . . . . . . 112

Глава 2. Конечномерные редукции в гладких экстремальных задачах на бесконечномерных многообразиях .......................... 114

$\S 2.1$.Редукция Ляпунова-Шмидта . . . . . . . . . . . . . . . . . . . . . . . . 115

$\S 2.2$. Редукция Морса-Ботта ...................................... 118

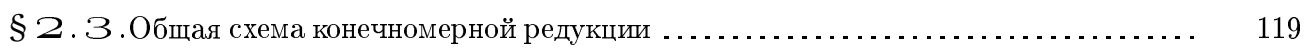

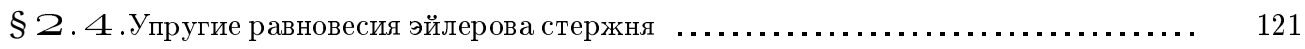

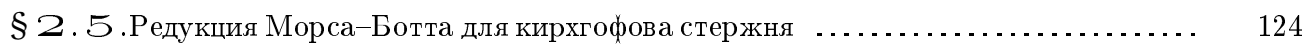

$\S 2.6$. Равновесия тонкой упругой пластины .............................. 127

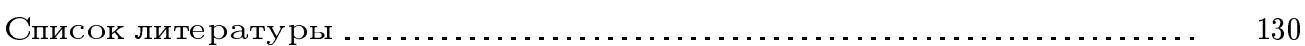

\section{Введение}

Широко известно, что многие нелинейные задачи математической физики допускают вариационную формулировку, то есть могут быть заданы в виде экстремальной задачи

$$
V(x) \rightarrow \text { inf }, \quad x \in X
$$

где $V$ - гладкий функционал на гладком банаховом многообразии $X$. Решение такой задачи часто можно осуществить переходом (редукцией) к аналогичной задаче

$$
W(\xi) \rightarrow \inf , \quad \xi \in Y
$$


где

$$
W(\xi)=\inf _{x: p(x)=\xi} V(x)
$$

$Y$ - гладкое конечномерное многообразие, $p$ - гладкая субмерсия $X \rightarrow Y$.

Данная статья посвящена описанию некоторых из наиболее используемых схем перехода от (0.1) к (0.2) при условии, что функция (0.3) наследует топологические и аналитические свойства функционала $V$ (сохраняются гладкость, количества и кратности критических точек, индексы Морса, гомотопические типы лебеговых множеств $\{V \leqslant c\}$ и т.д.). Среди требований, обеспечивающих выполнение этого условия, решаюшим является требование однозначности и гладкости отображения

$$
\varphi: \xi \mapsto\{x \in X: p(x)=\xi, W(\xi)=V(x)\} .
$$

Следуя [1], это отображение будем назьвать маргинальным. Функцию (0.3) будем назьвать ключевой, а отображение $p: X \rightarrow Y$ - редуцируюшим.

Основополагаюшими примерами конечномерных редукций являются локальная схема Ляпунова-Шмидта (в вариационной модификации) и глобальная схема Морса-Ботта из вариационной теории геодезических (см. [2]-[5] и [6], [7]). Эти схемы полезны прежде всего как инструменты для построения теории фредгольмовых особенностей гладких функционалов на банаховых многообразиях. В случае гильбертова многообразия такая теория была создана достаточно быстро [8], [9] (разумеется, на основе ранее созданной теории особенностей гладких функций конечного числа переменных). Казалось, что для получения аналогичной теории в случае банаховых многообразий достаточно лишш найти соответствуюшие варианты бесконечномерных банаховых обобщений леммы Морса и леммы о расщеплении особенности [9]-[11]. Однако результаты Н. А. Бобылева и Ю. М. Бурмана [12] показали, что возможности, открываемые банаховыми вариантами этих лемм, весьма ограниченны. По-видимому, в данной ситуации целесообразнее обратиться к прямым конечномерным редукциям.

Конечномерные редукции, основанные на редуцирующих субмерсиях и маргинальных отображениях, позволяют без существенных препятствий переносить конечномерную теорию особенностей гладких функций на случай фредгольмовых особенностей гладких функционалов.

Схемы конечномерных редукций представляют также прикладной интерес в качестве аппарата исследования потенциальных физических систем с неединственностью допустимых состояний. $\mathrm{K}$ таким системам относятся, например, упругие конструкции в закритических упруго равновесных состояниях [11], [13], [14]. Часто введением конечного набора дополнительных фиктивных связей можно добиться единственности допустимого устойчивого состояния (зависящего от параметров связей). В этом случае основные устойчивые состояния (выделяемые без учета дополнительных связей) могут быть найдены минимизацией по параметрам связей значений функционала энергии на множестве условно устойчивых состояний. Такой метод используется, например, в статике упругих стержней [13]. Сходные математические идеи применяются в теории нелинейных волн и некоторых других разделах нелинейной физики [15], [16]. В работах многих специалистов по нелинейному анализу высказьвалась общая мысль о том, что физические эффекты в системах с бесконечным числом 
степеней свободы часто допускают адекватное качественное описание через конечные наборы переменных - так называемые существенные, или поведенческие, переменные [11], [16]. Исторически схемам Ляпунова-Шмидта и Морса-Ботта предшествовал локальньй метод Пуанкаре понижения размерности в конечномерной экстремальной задаче [17], [18]. По-видимому, А. Пуанкаре предполагал, что его метод может быть применен и к системам с бесконечньм числом степеней свободы. Впервые математически строго обоснованные схемы редукций бесконечномерных систем к конечномерньм были предложены А. М. Ляпуновым [19] и Э. Шмидтом [20]. К сожалению, в работах [19], [20] и выросшей на их основе теории ветвления решений нелинейных уравнений [21] был утерян контекст вариационного происхождения. Возврат к вариашионньм корням произошел лишш в 70-е годы усилиями Н. А. Сидорова, В. А. Треногина, Н. А. Бобылева, М. А. Красносельского, Э. М. Мухамадиева, Дж. Марсдена и др. [2]-[5].

Впоследствии появились нелокальные версии схемы Ляпунова-Шмидта и ее новые применения в современных вариационных задачах [22]-[24].

Следует отметить также работы В. Койтера по анализу закритических равновесий упругих систем (см., например, [25]), в которых фактически использовалась (без математического обоснования) схема Ляпунова-Шмидта. В. Койтер отталкивался от упомянутого вьшше метода А. Пуанкаре и ритцевских конечномерных аптроксимаций.

Ритцевской аппроксимацией функционала $V$ на банаховом пространстве $E$ называется функция $W, W(\xi):=V\left(\sum_{j=1}^{n} \xi_{j} e_{j}\right), \xi=\left(\xi_{1}, \ldots, \xi_{n}\right)^{T}$, где $\left\{e_{1}, \ldots, e_{n}\right\}-$ некоторый линейно независимый набор функций (базис аппроксимации). Экстремалям $\bar{\xi}=\left(\bar{\xi}_{1}, \ldots, \bar{\xi}_{n}\right)^{T}$ функции $W$ соответствуют точки $\bar{x}=\sum_{j=1}^{n} \bar{\xi}_{j} e_{j}$, называемые ритцевскими аппроксимациями экстремалей $V$. Точность ритцевских аппроксимаций повьшшается за счет увеличения количества базисных функций. Если, обобщая, рассмотреть аппроксимации вида

$$
W(\xi)=V\left(\sum_{j=1}^{n} \xi_{j} e_{j}+\Phi(\xi)\right),
$$

где $\Phi$ - гладкое отображение из $N:=\operatorname{span}\left(e_{1}, \ldots, e_{n}\right)$ в $N^{\perp}$ (ортогональное дополнение к $N$ в метрике пространства функций с суммируемым квадратом), то во многих задачах можно достигнуть любой точности аппроксимации экстремалей при априори зафиксированном наборе базисных функций и, следовательно, априори ограниченном количестве степеней свободы аппроксимирующей системы. К аналогичным обобщениям можно прийти, отправляясь от конечно элементных аппроксимаций.

В данной статье изложены в современном виде схемы Пуанкаре, Ляпунова-Шмидта и Морса-Ботта с рассмотрением некоторых вариантов обобщений и примеров реализующих схем в конкретных задачах.

Автор глубоко благодарен В.И. Арнольду, Н.А. Бобылеву, Ю.Г. Борисовичу, П.П. Забрейко, В.Г. Звягину, М.А. Красносельскому, В.А. Кондратьеву, И. В. Скрыпнику, А. Т. Фоменко и В.В. Шарко за полезные высказывания и замечания по теме статьи, сделанные перечисленными математиками автору во время его выступлений на семинарах и в частных беседах. 
Работа вьполнена при финансовой поддержке международного научного фонда Сороса и Американского математического общества.

\section{Глава 1. Понижение размерности в конечномерных экстремальных задачах}

В данной главе дано описание редуцирующей схемы Пуанкаре с некоторыми обобщениями и примерами.

\section{§ 1.1. Редукция Пуанкаре}

Пусть задана гладкая экстремальная задача

$$
V(x) \rightarrow \inf , \quad x \in \mathbb{R}^{n},
$$

(гладкость означает принадлежность $V$ классу $C^{\infty}\left(\mathbb{R}^{n}\right)$ ). Если выполнено условие коэриитивности

$$
\lim _{|x| \rightarrow \infty} V(x)=\infty
$$

то задача (1.1.1) разрешима. Если, дополнительно, выполнено условие вьпуклости

$$
\frac{\partial^{2} V}{\partial x^{2}}(a)(h, h)>0 \quad \forall(a, h) \in \mathbb{R}^{n} \times\left(\mathbb{R}^{n} \backslash 0\right),
$$

то решение этой задачи является единственным.

Для построения приближенного решения задачи (1.1.1) при условиях (1.1.2) и (1.1.3) в вычислительной математике разработано много эффективных методов. Снятие условия (1.1.3) приводит к необходимости рассмотрения функций, имеющих несколько точек локальных минимумов. Поиск решения задачи (1.1.1) в этом случае сушественно затрудняется. Еще более сложной эта задача выглядит при наличии у функции $V$ дополнительных параметров, изменение которых приводит к бифуркационным явлениям. А. Пуанкаре предложил процедуру, позволяющую во многих случаях облегчить изучение ветвления экстремалей за счет исключения "несущественных" переменных.

Пусть выполнено условие выпуклости $V$ по части переменных:

$$
\frac{\partial^{2} V}{\partial x^{2}}(a)(h, h)>0 \quad \forall(a, h) \in \mathbb{R}^{n} \times(L \backslash 0),
$$

$L-l$-мерное подпространство в $\mathbb{R}^{n}$. Тогда вместо (1.1.1) можно рассмотреть аналогичную задачу с меньшим числом переменных

$$
W(\xi) \rightarrow \text { inf }, \quad \xi \in M,
$$

где $M$ - ортогональное дополнение к подпространству $L$ в $\mathbb{R}^{n}$ (в стандартной метрике $\left.(x, y)=\sum_{i=1}^{n} x_{i} y_{i}\right)$, а $W$ - функция, определенная соотношением

$$
W(\xi)=\inf _{h: h \in L} V(\xi+h) .
$$

ТЕОРема 1.1.1. При выполнении условий (1.1.2) и (1.1.4) функция (1.1.6) является гладкой. 
ДокаЗАтельство. Решение задачи $V(\xi+h) \rightarrow \inf , h \in L$, задается системой уравнений

$$
\frac{\partial V}{\partial e_{j}}(\xi+h)=0, \quad j=1, \ldots, l,
$$

где $\left\{e_{1}, \ldots, e_{n}\right\}-$ произвольньй фиксированньй базис в $L$. Якобиан левой части этой системы является невырожденным вследствие (1.1.4). Поэтому гладкость решения $h=h(\xi)$ системы уравнений (1.1.7) обеспечивается теоремой о неявной функции. Существование и единственность решения обеспечивается условиями (1.1.2) и (1.1.4). Следовательно, функция $W(\xi)=V(\xi+h(\xi))$ является гладкой.

ЗАмечание 1.1.1. Строго говоря, А. Пуанкаре определял функцию $W$ не формулой (1.1.6), а системой уравнений (1.1.7). Функция $W$ при этом определялась локально (условия (1.1.2) не было) и предназначалась для локального исследования бифуркации экстремалей [17], [18]. Смысл перехода от (1.1.1) к (1.1.5) состоял в замене многомерной задачи на маломерную.

ОПРЕДЕЛЕНИЕ 1.1.1. Функция (1.1.6), полученная при условиях (1.1.2) и (1.1.4), назьвается ключевой. Координаты $\xi_{j}, j=1, \ldots, m=n-l$, вектора $\xi \in M$ относительно произвольного фиксированного базиса $f_{1}, \ldots, f_{m}$ в $M$ также называются ключевыми. Отображение $\varphi: \xi \mapsto \xi+h(\xi)$, где $h(\xi)$ определено системой уравнений (1.1.7), называется марәинальныц.

Теорема 1.1.2. Маргинальное отображение задает взаимно однозначное соответствие между множеством критических точек функиии $V$ и множеством критических точек ее ключевой функиии $W$. При этом соответствии невырожденные критические точки переходят в невырожденные и только невырожденные критические точки с сохранением значений индексов Морса. А соответствующие друг другу вырожденные критические точки имеют изоморфные локальные кольца особенностей ${ }^{1}$.

ДОКАЗАТЕЛЬСТВО становится очевидным после применения обобщенной леммы Морса (леммы о расщеплении особенности) [11], [26].

ОПРЕДЕЛЕНИЕ 1.1.2. Переход от задачи (1.1.1) к задаче (1.1.5), где $W$-ключевая функция $V$, назьвается редукиией по схеме Пуанкаре или, более кратко, редукиией Пуанкаре.

ЗАмЕчАнИЕ 1.1.2. В формулировках теорем 1.1.1 и 1.1.2 условие (1.1.1) можно ослабить, заменив его условием коэриитивности по компоненте $h \in L$.

\footnotetext{
${ }^{1}$ Локальным кольцом особенности гладкой функции $V$ в критической точке $a \in \mathbb{R}^{n}$ назьвается фактор $\mathscr{E}_{a}\left(\mathbb{R}^{n}\right) / \mathfrak{A}$ кольца ростков $\mathscr{E}_{a}\left(\mathbb{R}^{n}\right)$ гладких функций на $\mathbb{R}^{n}$ в точке $a$ по якобиеву идеалу $\mathfrak{A}$, порожденному ростками функций $\frac{\partial V}{\partial x_{j}}, j=1, \ldots, n,[11],[26]$.
} 


\section{§1.2. Обобщенная редукция Пуанкаре}

Описанная в $\S 1.1$ схема допускает естественное обобщение, основанное на замене линейного отображения $p: X \rightarrow \mathbb{R}^{m}$ гладкой субмерсией.

Пусть $V$-гладкая функция на $\mathbb{R}^{n}$ и пусть задано гладкое отображение $p: \mathbb{R}^{n} \rightarrow \mathbb{R}^{m}$ с условием $\mathrm{rk} \frac{\partial p}{\partial x}(a)=m \forall a \in \mathbb{R}^{n}$. Пусть область $\mathscr{O}$ является образом $p$ в $\mathbb{R}^{m}(\mathscr{O}-$ открытое связное подмножество в $\left.\mathbb{R}^{m}\right)$. Предположим, что выполняются следуюшие два условия:

А) для каждой точки $\xi \in \mathscr{O}$ подмногообразие $p^{-1}(\xi)$ в $\mathbb{R}^{n}$ является геодезически выпуклым м, то есть для любой пары точек $a, b \in p^{-1}(\xi)$ сушествует единственная геодезическая кривая на $p^{-1}(\xi)$ (в индуцированной из $\mathbb{R}^{n}$ метрике), соединяюшая эти точки;

В) для любой точки $\xi \in \mathscr{O}$ функция $\left.V\right|_{p^{-1}(\xi)}$ является геодезически строго виnуклой: для любой геодезической кривой $x=x(t), t \in(\alpha, \beta)$, на $p^{-1}(\xi)$ функция $\omega(t)=V(x(t))$ является строго выпуклой, то есть $\ddot{\omega}(s)>0 \forall s \in(\alpha, \beta)$.

Рассмотрим функцию $W$, определенную формулой

$$
W(\xi)=\inf _{x: p(x)=\xi} V(x)
$$

ТеОРема 1.2.1. При въполнении для гладкой коэриитивной функиии V условий А) и В) функиия $W$, определенная соотношением (1.2.1), является гладкой.

ДокАЗАтЕльство. Из коэрцитивности $V$ на $\mathbb{R}^{n}$ вытекает ограниченность $V$ снизу. Следовательно, $W(\xi)>-\infty \forall \xi \in \mathscr{O}$. Более того, для каждого $\xi$ найдется единственная точка $\varphi(\xi) \in p^{-1}(\xi)$, для которой $W(\xi)=V(\varphi(\xi))$ (это следует из коэрцитивности и выпуклости $\left.\left.V\right|_{p^{-1}(\xi)}\right)$. Если $x=x(t)$ - геодезическая на $p^{-1}(\xi)$, выходяшая из точки $\varphi(\xi)$ с произвольно заданной скорость $h \neq 0$, то для соответствующего приращению $h$ значения второго дифференциала сужения $\left.V\right|_{p^{-1}(\xi)}$, вычисленного в точке $\varphi(\xi)$, имеем

$$
\frac{\partial^{2}}{\partial x^{2}}\left(\left.V\right|_{p^{-1}(\xi)}\right)(\varphi(\xi))(h, h)=\left.\frac{d^{2}}{d t^{2}}(V(x(t)))\right|_{t=0}>0
$$

(см. условие В)). Из этого соотношения вытекают невырожденность гессиана $\left.V\right|_{p^{-1}(\xi)}$ в точке $\varphi(\xi)$ и, следовательно, гладкая зависимость $\varphi(\xi)$ от $\xi$ (в силу теоремы о неявной функции). Следовательно, функция (1.2.1) является гладкой как композиция гладкой функции $V$ и гладкого маргинального отображения

$$
\xi \mapsto \varphi(\xi) .
$$

ОПРЕДЕЛЕНИЕ 1.2.1. Функция (1.2.1), определенная при условиях А) и В), называется ключевой, а субмерсия $p: \mathbb{R}^{n} \rightarrow \mathscr{O}$ назьвается редущирующим отображениe⿻从. 
ТЕОРема 1.2.2. В условиях теоремь 1.2.1 маргинальное отображение (1.2.2) устанавливает взаимно однозначное соответствие между множеством критических точек функиии $V$ и множеством критических точек ключевой функиии (1.2.1). При этом соответствующие друг другу критические точки имеют изоморфные локальные кольца особенностей и, в случае невырожденности критических точек, равные индексы Морса.

ДоКАЗАТЕЛЬСТво (его решаюший шаг) состоит в непосредственном применении леммы о расщеплении особенности [11], [26].

Описанная схема естественно распространяется на гладкие функции, заданные на полном римановом многообразии $\mathscr{M}$. Условие коэрцитивности $V$ формулируется при этом следуюшим образом: если $\left\{a_{k}\right\}_{k=1}^{n}$ - не предкомпактная последовательность точек $\mathscr{M}$, то $\sup \left\{V\left(a_{k}\right)\right\}=\infty$.

Образом редуцирующего отображения также может быть гладкое многообразие $\mathscr{N}$.

Разумеется, сушествуют и другие варианты обобшения. Например, можно рассмотреть функцию $V$ на римановом многообразии $\mathscr{M}$ с гладкой субмерсией $p: \mathscr{M} \rightarrow \mathcal{N}$ при условии существования такого значения $c$ для $V$, что

А) множество $p^{-1}(\xi) \cap\{V \leqslant c\}$ компактно и геодезически вьпукло $\forall \xi \in \mathscr{N}$,

В) для каждого $\xi \in \mathscr{N}$ функция $\left.V\right|_{p^{-1}(\xi) \cap\{V \leqslant c\}}$ геодезически строго выпукла.

Если не задаваться целью указьвать конкретные условия, гарантирующие однозначность маргинального отображения, то можно формулировать обшие принципы редукции, подобные теоремам редукции Морса-Ботта [7] .

ТЕОРема 1.2.3. Пусть на гладком не имеющем края многообразии М задана гладкая функиия $V$, для которой

1) существует такое регулярное значение $c$, что многообразие $\{V \leqslant c\}^{2}$ компактно;

2) существует гладкая субмерсия $p:\{V \leqslant c\} \rightarrow \mathscr{N}$, где $\mathscr{N}-$ гладкое многообразие (вообще говоря, с краем), для которой $p^{-1}(\xi)$ имеет непустое пересечение с внутренностью множества $\{V \leqslant c\} \quad \forall \xi \in \mathscr{N} \backslash \partial \mathscr{N}$, это пересечение содержит единственную (в нем) критическую точку $\varphi(\xi)$ для $\left.V\right|_{p^{-1}(\xi)}$, и точка $\varphi(\xi)$ является морсовской точкой минимума для $\left.V\right|_{p^{-1}(\xi)}$

3) если $\partial \mathscr{N} \neq \varnothing$, mо $p^{-1}(\partial \mathscr{N}) \subset\{V=c\}$, и любая точка $a \in p^{-1}(\partial \mathscr{N})$ является морсовской критической точкой $\left.д л я ~ V\right|_{\widetilde{p}^{-1}(\xi)}, \xi=p(a)$, где $\widetilde{p}$ - любое гладкое продолжение отображсния р на любую окрестность $\mathscr{O}$ точки а в $\mathscr{M}$ с множества $\mathscr{O} \cap\{V \leqslant c\}$.

Тогда

1) функция $W, W(\xi):=\inf _{x: x \in p^{-1}(\xi) \cap\{V \leqslant c\}} V(x)$, является гладкой;

2) отображение $\xi \mapsto \varphi(\xi)$ является гладким и устанавливает взаимно однозначное соответствие между множеством критических точек $V$ в $\{V \leqslant c\}$ и множеством критических точек $W$ в $\mathscr{N} \backslash \partial \mathscr{N} ;$

\footnotetext{
${ }^{2}\{V \leqslant c\}$ - краткое обозначение лебегова множества $\{x \in \mathscr{M}: V(x) \leqslant c\}$.
} 
3) в соответствующих друг другу критических точках локальные кольца особенностей функиий $V$ и $W$ изоморфнь;

4) индексы Морса соответствующих друг другу невырожсенных критических точек совпадают;

5) отображсения $p:\{V \leqslant c\} \rightarrow \mathscr{N} u \varphi: \mathscr{N} \rightarrow\{V \leqslant c\}$ гомотопически обратны друг другу (их композиции гомотопны единицам: $\varphi \circ p \sim I, p \circ \varphi \sim I)$.

ДокАЗАТЕЛЬСТво. Из условий 1), 2) следует, что $\varphi(\xi)$ - точка минимума для $\left.V\right|_{p^{-1}(\xi)} \forall \xi \in \mathscr{N} \backslash \partial \mathscr{N}$, и отображение $\varphi$ вместе с функцией $W=V \circ \varphi$ являются гладкими.

Доказательства утверждений 3), 4) являются повторениями доказательств аналогичных утверждений теоремы 1.1.2 (после выбора подходящих локальных координат в окрестностях рассматриваемых точек).

Так как $p(\varphi(\xi)) \equiv \xi$, то проверка утверждения 5) сводится к проверке гомотопности единице отображения $\varphi$ о . Но данная гомотопия осушествляется простым стягиванием в точки $\varphi(\xi)$ слоев по линиям кратчайшего спуска: вдоль интегральных кривых векторного поля $-\operatorname{grad}\left(\left.V\right|_{p^{-1}(\xi)}\right)$ (градиент определен по какой-либо фиксированной римановой метрике на $\{V \leqslant c\})$.

ПримеР 1.2.1. Пусть двумерный тор $T^{2}$ “вертикально стоит" в $\mathbb{R}^{3}$ на плоскости переменных $\{x, y\}$ так, что меридианы тора параллельны плоскости переменных $\{x, z\}$ (рис. 1.2.1). Пусть $V$ - функция высоты: $V(x, y, z)=z \forall(x, y, z) \in T^{2}$. Если $c_{1}<$ $c_{2}<c_{3}<c_{4}$ - критические значения $V$ на $T^{2}$, то для любого $c \in\left(c_{1}, c_{2}\right)$ множество $\{V \leqslant c\}$ можно рассматривать как график некоторой гладкой функции $z=\psi(x, y)$ на области $\mathscr{D}$, являющейся образом ортогональной проекции множества $\{V \leqslant c\}$ на плоскость переменных $\{x, y\}$. Функция $V$ на $\{V \leqslant c\}$ допускает редукцию к функции одной переменной $W$ :

$$
W(\xi):=\inf _{y:(\xi, y) \in \mathscr{D}} \psi(\xi, y)=\psi(\xi, 0), \quad|\xi| \leqslant d
$$

где $d=\max \{x: \psi(x, y)=c\}$

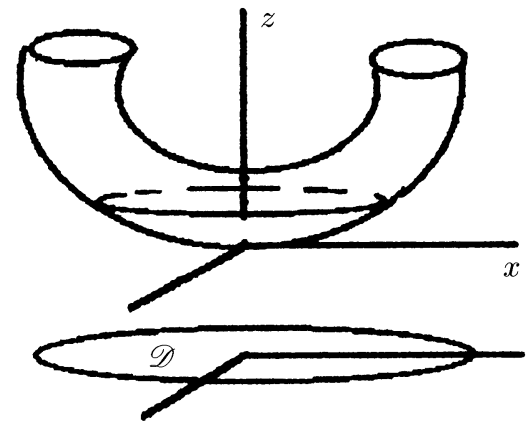

Рис. 1.2.1 
Редуцируюшая субмерсия и маргинальное отображение здесь задаются, соответственно, действиями $(x, y, z) \mapsto x$ и $\xi \mapsto(\xi, 0, \psi(\xi, 0))$.

В этом же примере можно осуществить другую редукцию. Введем на $T^{2}$ угловые координаты $(\alpha, \beta)$, где $\alpha$ - угол вдоль параллели, отсчитываемый от самого "большого" меридиана до заданной точки $\tau$, а $\beta$ - угол вдоль меридиана, отсчитьваемый от самой нижней параллели. Положим $\widetilde{p}(\tau)=\alpha$. Субмерсия $\widetilde{p}$ осушествляет редукцию $V$ на области $\mathscr{M}=\left\{\tau \in T^{2}:|\beta|<\pi\right\}$ к функции высоты $\widetilde{W}$ на окружности $S^{1}=\{z \in \mathbb{C}:|z|=1\}$, канонически отождествленной с нижней параллелью. Маргинальное отображение $\varphi: S^{1} \rightarrow \mathscr{M}$ является отображением естественного вложения параллели в тор. Вторая редукция является “более удачной”, так как она осушествлена на более широкой области и охватьвает две критические точки, расположенные на многообразиях уровней $\left\{V=c_{1}\right\}$ и $\left\{V=c_{2}\right\}$.

ПримеР 1.2.2. Среди аналитических конструкций, укладывающихся в схему Пуанкаре, по степени известности выделяется преобразование Лежандра. В классической механике оно осушествляет связь между лагранжевым и гамильтоновым формализмами [27]: если $L(\dot{q}, q, t)$ - лагранжиан какой-либо механической системы, то ее гамильтониан $H(p, q, t)$ получается из $L$ преобразованием Лежандра:

$$
H(p, q, t)=\sup _{\dot{q}}((\dot{q}, p)-L(\dot{q}, q, t))=-\inf _{\dot{q}}(L(\dot{q}, q, t)-(\dot{q}, p)), \quad \dot{q}, p, q \in \mathbb{R}^{n}
$$

С точки зрения редукции Пуанкаре соотношение (1.2.3) исключает переменную $\dot{q}$ в функции $V, V(\dot{q}, p, q, t):=L(\dot{q}, q, t-(\dot{q}, p)$. Лагранжиан получается из гамильтониана повторным применением преобразования Лежандра.

В учебниках по аналитической механике обычно приводится лишш условие локальной редуцируемости, заключаюшееся в требовании выпуклости лагранжиана по переменной $\dot{q}$. Коэрцитивность же $V$ по переменной $\dot{q}$ в реальных механических системах автоматически обеспечивается квадратичной зависимостью кинетической энергии от вектора скорости. После перехода к обобщенным координатам коэрцитивность $V$ по вектору скорости сохраняется.

Общая механическая система, в которой соответствующее маргинальное отображение $\varphi:(p, q, t) \rightarrow(\dot{q}, p, q, t)$ глобально определяется соотношением (1.2.3), называется системой, удовлетворяюшей условию двойственности Лагранжа-Гамильтона [28].

\section{§1.3. Посткритические равновесия упругой цепи}

Пусть дана система одинаковых абсолютно твердых прямолинейных стержней длины единища, последовательно соединенных одинаковыми упругими шарнирами в прямолинейную $(n+1)$-звенную упругую цепь (рис. 1.3.1). Предположим, что первое и последнее звенья закреплены так, что они остаются в вертикальном положении при всех деформациях цепи. Пусть $\varphi_{j}-$ угол между $j$-м звеном и вертикалью, $j=1, \ldots, n+1$, образованньй в результате деформации цепи под воздействием продольно сжимаюшей нагрузки. Если принять соглашение о том, что упругие шарниры 
"работают" по закону Гука (момент силы упругой реакции пропорционален углу между звеньями), то равновесная конфигурация цепи определится системой уравнений

$$
\left\{\begin{array}{l}
\varphi_{j+1}-2 \varphi_{j}+\varphi_{j+1}+\lambda \sin \varphi_{j}=0, \quad j=2, \ldots, n \\
\varphi_{1}=\varphi_{n+1}=0
\end{array}\right.
$$

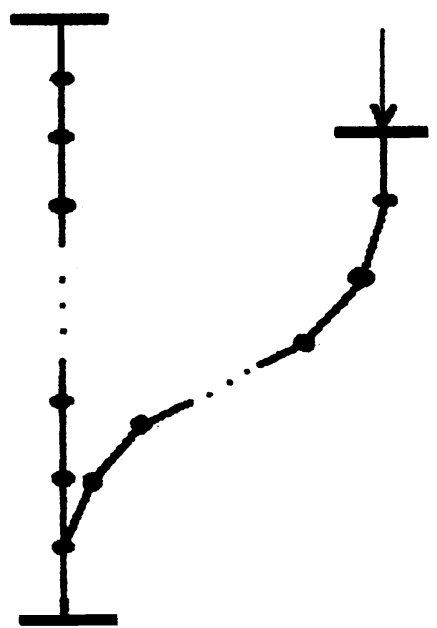

Рис. 1.3.1

Параметр $\lambda$ в этих уравнениях пропорционален величине сжимаюшей силы. Масштаб длины предполагается выбранным здесь так, что соответствующее произведение входяших в уравнение упруго-механических и геометрических констант равно единице.

Система уравнений (1.3.1) потенциальна: ее левая часть состоит из компонент градиента функции полной энергии

(1.3.2) $V(\varphi, \lambda)=\sum_{j=1}^{n}\left(\frac{\left(\varphi_{j+1}-\varphi_{j}\right)^{2}}{2}+\lambda\left(\cos \varphi_{j}-1\right)\right)=\frac{1}{2}(A \varphi, \varphi)+\lambda \sum_{j=2}^{n}\left(\cos \varphi_{j}-1\right)$

(с учетом соотношений $\left.\varphi_{1}=\varphi_{n+1}=0\right)$, где $\varphi=\left(\varphi_{2}, \ldots, \varphi_{n}\right)^{T}$ и

$$
A=\left(\begin{array}{cccccc}
2 & -1 & 0 & & & \\
-1 & 2 & -1 & \ddots & & \\
0 & -1 & 2 & \ddots & & \\
& \ddots & \ddots & \ddots & & \\
& & & & 2 & -1 \\
& & & & -1 & 2
\end{array}\right) .
$$


Функция (1.3.2) отличается лишь постоянным множителем от разностной аппроксимации функционала полной энергии продольно сжатого упругого эйлерова стержня с жестко заделанными концами [13]. Связь с эйлеровым стержнем проявляется и в спектральных характеристиках матришы $A$. Действительно, собственными значениями $A$ являются числа

$$
\lambda_{k}=2\left(1-\cos \left(\frac{\pi k}{n}\right)\right), \quad k=1, \ldots, n-1,
$$

которым отвечают собственные векторы

$$
g_{k}=\left(\alpha_{1}^{k}, \ldots, \alpha_{n-1}^{k}\right)^{T}, \quad \alpha_{j}^{k}=\sin \left(\frac{\pi k j}{n}\right) .
$$

Нетрудно заметить, что $\lim _{n \rightarrow \infty} n^{2} \lambda_{k}=(\pi k)^{2}$, то есть $k$-е собственное значение матришы $n^{2} A$ при $n \rightarrow \infty$ стремится к $k$-му собственному значению оператора $-\frac{d^{2}}{d s^{2}}$ на отрезке $[0,1]$ (при краевых условиях $x(0)=x(1)$ ). Этому собственному значению оператора отвечает собственная функция $x_{k}(s)=\sin (\pi k s)$. Ее значения в узлах $s=j / n$ являются координатами собственного вектора $g_{k}$ матришы $A$.

Если $\lambda<\lambda_{1}$, то

$$
\frac{\partial^{2} V}{\partial \varphi^{2}}(\varphi, \lambda)(h, h)=(A h, h)-\lambda \sum_{j=2}^{n}\left(\cos \varphi_{j}\right) h_{j-1}^{2} \geqslant\left(\lambda_{1}-\lambda\right)|h|^{2},
$$

то есть при $\lambda<\lambda_{1}$ функция $V$ вьпукла по $\varphi$.

Коэрцитивность $V$ очевидна.

Следовательно, при $\lambda<\lambda_{1}$ точка $\varphi=0$ является единственной критической точкой $V$, и она же является точкой глобального минимума $V$.

Если задано ограничение $\lambda<\lambda_{m+1}$, то можно гарантировать лишш следуюшее неравенство

$$
\frac{\partial^{2} V}{\partial \varphi^{2}}(\varphi, \lambda)(h, h)>0 \quad \forall h \in L_{m} \backslash 0,
$$

где $L_{m}=\operatorname{span}\left(g_{1}, \ldots, g_{m}\right)$.

Положим $e_{k}=g_{k} /\left|g_{k}\right|$. В соответствии с редуцируюшей схемой Пуанкаре можно перейти к ключевой функции $W$

$$
W(\xi, \lambda):=\inf _{h: h \in L_{\frac{1}{m}}^{\perp}} V\left(\sum_{j=1}^{m} \xi_{j} e_{j}+h, \lambda\right), \quad \xi \in \mathbb{R}^{m},
$$

"отвечающей" за поведение $V$.

Приближенное вычисление $W$ и дальнейший ее анализ требуют привлечения компюютера. Если же ограничиться локальной задачей анализа ветвления экстремалей 
в окрестности нуля, то с помошью специальных асимптотических методов можно достигнуть достаточно полного результата "вручную". Например, при $m=1$ можно воспользоваться представлением

$$
W(\xi, \lambda)=V\left(\xi e_{1}, \lambda\right)+o\left(\xi^{5}\right)+O\left(\lambda-\lambda_{1}\right) O\left(\xi^{4}\right)
$$

легко проверяемым, если учесть четность $V(\cdot, \lambda)$ и представимость маргинального отображения в виде $\varphi(\xi, \lambda)=\xi e_{1}+o\left(\xi^{2}\right)$.

Следовательно,

$$
W(\xi, \lambda)=\alpha(\lambda) \frac{\xi^{2}}{2}+a^{2} \lambda_{1} \frac{\xi^{2}}{24}+o\left(\xi^{5}\right)+O\left(\lambda-\lambda_{1}\right) O\left(\xi^{4}\right)
$$

где $\alpha(\lambda)=\lambda_{1}-\lambda, a^{2}=\gamma_{1}^{4}+\cdots+\gamma_{n-1}^{4}, \gamma_{j}-j$-я компонента вектора $e_{1}$.

Из теории нормальных форм особенностей гладких функций и их версальных деформаций [26], [11] следует - в случае представимости в форме (1.3.3) - приводимость функции $W$ к виду $\frac{\alpha(\lambda)}{2} \xi^{2}+\frac{a^{2} \lambda}{24} \xi^{4}$ посредством замены $\xi \mapsto \xi+o\left(\xi^{2}\right)$ (при $\lambda$, достаточно близком к $\left.\lambda_{1}\right)$. Таким образом, поведение функции $V(\cdot, \lambda)$ в окрестности нуля при малых $\lambda-\lambda_{1}$ определяется главной частью ее ключевой функции (с одной ключевой переменной).

ЗАМЕчАнИЕ 1.3.1. Изучение характера ветвления экстремалей и вычисление первых асимптотик ветвей бифурцируюших экстремалей по закритическому прирашению $\delta=\lambda-\lambda_{1}$ можно осуществить, применив к функции (1.3.3) масштабирующее преобразование (преобразование Вейерштрасса, $\sigma$-процесс) $\xi=\delta^{1 / 2} \eta$ с последующим выделением главной части получаемой функции и применением теоремы о неявной функции.

\section{§ 1.4. Стационарные врашения} многомерных волчков. Редукция Смейла

Известно, что устойчивость стационарного вращения твердого тела вокруг оси, проходящей через точку опоры и центр тяжести (в режиме спящего волчка), определяется неравенством $C \omega^{2}>4 A m g l$ - в случае динамической симметрии, и неравенством $\min \{C-A, C-B\} \omega^{2}>m g l$ - для несимметричных волчков [27], [29]. Здесь $A, B, C$ - главные моменты инерции тела, $\omega$ - угловая скорость вращения, $l$ - расстояние от точки опоры до центра тяжести, $m g$ - вес тела.

Бифуркашии врашений твердого тела, возникающие в результате потери устойчивости стационарного вращения, описаны в монографии В. Н. Рубановского и В. А. Самсонова [29].

Один из современных способов, которым можно изучать устойчивость и бифуркации стационарных вращений несимметричного волчка, состоит в использовании эффективного потенциала, профакторизованного по действию группы симметрии. В результате факторизации получается функция на двумерной сфере. Ее точки минимума соответствуют устойчивым врашениям [27], [29], [30]. 
В случае многомерного волчка [27], [31]-[31.8] факторизация эффективного потенциала по орбитам группы симметрии не приводит к функции на сфере. Полученная функция зависит еще от дополнительных переменных, отвечающих за расположение подгруппы симметрий относительного кинетического момента в группе ортогональных преобразований, сохраняющих вертикаль [32], [33]. Если значение относительного кинетического момента регулярно (его аннулятор - подалгебра Картана), то эта подгруппа - максимальньй тор в группе ортогональных преобразований, сохраняющих вертикаль. Дополнительные переменные можно исключить по редуцирующей схеме Пуанкаре. После исключения получается функция на сфере, критические точки которой соответствуют стационарным врашениям.

Движение $n$-мерного тела в поле тяготения задается обобшенными уравнениями Эйлера-Пуассона на группе $\mathrm{SO}(n)[27],[31]-[31.7]$ (очерк по истории исследований динамики $n$-мерного твердого тела имеется в [31.8]):

$$
\left\{\begin{array}{l}
M \dot{\Omega}+\dot{\Omega} M+[\Omega, M \Omega+\Omega M]+\lambda r \wedge \gamma=0 \\
\dot{\gamma}+\Omega \gamma=0
\end{array}\right.
$$

в которых $\Omega=f^{-1} \frac{d f}{d s}-$ угловая скорость (в теле), $f(t)$ - функция со значениями в $\mathrm{SO}(n), M=\operatorname{diag}\left(m_{1}, \ldots, m_{n}\right), m_{j}>0, \lambda$ - параметр величины силы тяжести, $\gamma=f^{-1} e_{n}$-вектор Пуассона, $e_{n}=(0, \ldots, 0,1)^{T}$ - вертикальньй орт. Через $[X, Y]$ обозначается коммутатор матриц $X, Y \in \mathrm{so}(n)$, а через $r \wedge \gamma$ - бивектор (кососимметрическая матриша, определенная соотношением $(r \wedge \gamma) x=(r, x) \gamma-(\gamma, x) r)$. Постоянный вектор $r$ обозначает центр тяжести.

Система двух уравнений (1.4.1) сводится к одному уравнению

$$
M \dot{\Omega}+\dot{\Omega} M+\left[\Omega^{2}, M\right]+\lambda r \wedge f^{-1} e_{n}=0 .
$$

Пусть $E(f, \Omega)=K(\Omega, \Omega)+U(f)$ - полная энергия волчка, $K=(\Omega, \Omega):=\frac{1}{2}\langle\mathscr{M}(\Omega), \Omega\rangle$ - кинетическая энергия волчка $\left(\mathscr{M}(\Omega):=M \Omega+\Omega M,\langle X, Y\rangle:=\frac{1}{2} \operatorname{tr} X^{T} Y\right), U(f):=$ $\lambda(r, \gamma)$ - потенциальная энергия поля тяготения. Отображение относительного кинетического момента $p:(f, \Omega) \mapsto \pi J(f, \Omega)$, где $J(f, \Omega)=f \mathscr{M}(\Omega) f^{-1}, \pi$ - ортопроектор в метрике $K(X, Y)$ из $\operatorname{so}(n)$ на $\mathfrak{N}:=\operatorname{Ann}\left(e_{n}\right)=\left\{Y \in \operatorname{so}(n): Y e_{n}=0\right\}$, является интегралом уравнения (1.4.2) (см. [32]), а соответствуюшее интегральное множество $F_{p}$ является гладким подмногообразием размерности $(n-1)(n+2) / 2 \forall p \in \mathfrak{N}$. Кроме того, $F_{p}$ - подрасслоение тривиального расслоения $\mathrm{SO}(n) \times \mathrm{so}(n) \rightarrow \mathrm{SO}(n)$ со слоем $F_{p, f}=\{\Omega \in \operatorname{so}(n): p(f, \Omega)=p\} \quad \forall f \in \mathrm{SO}(n)$. Множество $F_{p}$ инвариантно относительно действия $(g,(f, \Omega)) \rightarrow(g f, \Omega), f \in G_{p}$, где $G_{p} \subset \mathrm{SO}(n-1)$ - подгруппа изотропии в точке $p$ действия $(g, p) \mapsto g p g^{-1}(\mathrm{SO}(n-1)$ отождествлена с подгруппой $\left.\left\{u \in \operatorname{SO}(n): u e_{n}=e_{n}\right\}\right)$.

Стационарные врашения соответствуют критическим орбитам действия $G_{p}$ на $F_{p}$ относительно $E(f, \Omega)[34],[26]$. Их можно разыскивать посредством редукции Смейла к приведенному потенциалу [34]:

$$
U_{p}(f)=\min _{\Omega: \Omega \in F_{p, f}} E(f, \Omega) .
$$


Так как $E(f, \Omega)$ выпукла и квадратично зависит от $\Omega$, то для нее вьполняются условия А) и В) $\S 1.2$. Следовательно, сушествует гладкое (по $(f, p))$ маргинальное отображение $h_{p}: \mathrm{SO}(n) \rightarrow F_{p}$, для которого $U_{p}(f)=E\left(f, h_{p}(f)\right)$. Нетрудно проверить, что $h_{p}\left(g^{-1} f\right)=h_{g p g^{-1}}(f) \forall g \in \mathrm{SO}(n-1)$. Следовательно, $U_{p}\left(g^{-1} f\right)=U_{g p g^{-1}}(f)$.

Таким образом, $U_{p}(f)$ допускает факторизацию:

$$
U_{p}(f)=\widehat{U}_{p}([f]), \quad[f] \in \operatorname{SO}(n) / G_{p}
$$

В дальнейшем будем предполагать, что $p \in \operatorname{Reg}(\mathfrak{N})$ (то есть $\operatorname{Ann}(p)$ - подалгебра Картана в $\mathfrak{N}$ ).

Действие (левое) $\mathrm{SO}(n-1) \times \mathrm{SO}(n) \rightarrow \mathrm{SO}(n)$ порождает действие

$$
\mathrm{SO}(n-1) \times\left(\mathrm{SO}(n) / G_{p}\right) \rightarrow\left(\mathrm{SO}(n) / G_{p}\right),
$$

все орбиты которого имеют общий диффеоморфный тип, равный $\mathrm{SO}(n-1) / G$.

Пусть выполнены следующие два условия:

1) задана такая окрестность $\mathscr{O}$ единицы в $\mathrm{SO}(n)$, что пересечение с $\mathscr{O}$ каждой орбиты действия (1.4.3) содержит не более одной критической точки сужения потенциала $\widehat{U}_{p}$ на эту орбиту;

2) критические точки сужений $\widehat{U}_{p}$ на пересечения $\mathscr{O}$ с орбитами действия (1.4.3) являются морсовскими точками минимумов.

Неравенства, которым должны подчиняться $p$ и $M$ при вьполнении данных требований, приведены в [32], [33].

Анализ бифуркации стационарных врашений сводится при этих условиях к анализу бифуркации экстремалей ключевой функции $W$

$$
W(\xi):=\inf _{f: \pi(f)=\xi} U_{p}(f), \quad \xi \in S^{n-1}
$$

на сфере $S^{n-1}=\left\{x \in \mathbb{R}^{n}:|x|=1\right\}$. Через $\pi$ здесь обозначено каноническое отображение $\mathrm{SO}(n) \rightarrow S^{n-1}, \pi(f):=f e_{n}$. Локальньй анализ функции $W$ проведен в [32], [33]. Там же приведены условия устойчивости стационарного вращения при $\gamma=r=e_{n}, n=2 k+1$ (в четномерном пространстве спящих волчков нет).

\section{Глава 2. Конечномерные редукции в гладких экстремальных задачах на бесконечномерных многообразиях}

В данной главе дано описание бесконечномерных аналогов редуцирующей схемы Пуанкаре. Основной упор сделан на схемы Ляпунова-Шмидта и Морса-Ботта. Бесконечномерность вносит свой специфический аспект в проблему, связанньй с тем, что одна и та же экстремальная задача может рассматриваться в различных функциональных пространствах с различными геометрическими и топологическими свойствами. Задачи, в которых допускаются редуцируюшие переходы к конечномерным экстремальным задачам, в некотором смысле инвариантны по отношению к смене функциональных пространств, так как за топологические свойства соответствующих функционалов отвечают функции (ключевые) на конечномерных подмногообразиях. 


\section{§ 2.1. Редукция Ляпунова-Шмидта}

Пусть $f: E \rightarrow F$-гладкое фредгольмово нулевого индекса отображение банаховых пространств [16], [21], [35]. Пусть $f$ собственно (прообраз любого компакта компактен) и потенциально:

$$
\langle f(x), h\rangle \equiv \frac{\partial V}{\partial x}(x) h,
$$

где $V$ - гладкий функционал на $E$ (потенциал отображения $f),\langle\cdot, \cdot\rangle$ - скалярное произведение в некотором гильбертовом пространстве $H$, содержашем $E$ и $F$ как непрерывно и плотно вложенные подпространства. Предполагается также, что $E$ непрерывно вложено в $F$.

Если выполнено условие положительности (монотонности)

$$
\left\langle\frac{\partial f}{\partial x}(x) h, h\right\rangle>0 \quad \forall(x, h) \in E \times(E \backslash 0),
$$

то уравнение

$$
f(x)=0
$$

однозначно разрешимо. Это верно в силу теоремы Банаха-Каччиополи-Мазура [36], $[35],[37]$. Очевидно, что решение этого уравнения является и точкой глобального минимума $V$ на $E$.

Уравнение (2.1.3) - абстрактньй аналог уравнения Эйлера-Лагранжа из вариационного исчисления.

Если (2.1.2) заменить более слабым условием

$$
\left\langle\frac{\partial f}{\partial x}(x) h, h\right\rangle>0 \quad \forall(x, h) \in E \times\left(R^{*} \backslash 0\right),
$$

где $R^{*}=E \cap N^{\perp}, N=\operatorname{span}\left(e_{1}, \ldots, e_{n}\right), N^{\perp}$ - ортогональное дополнение к $N$ в $H,\left\{e_{1}, \ldots, e_{n}\right\}$ - некоторая ортонормированная в $H$ система векторов в $E$, то можно определить ключевую функцию $W$

$$
W(\xi):=\inf _{x:\left\langle x, e_{j}\right\rangle=\xi_{j} \forall j} V(x), \quad \xi=\left(\xi_{1}, \ldots, \xi_{n}\right)^{T},
$$

“отвечающую” за поведение функционала $V$. Условие собственности $f$ можно ослабить, заменив его условием собственности при каждом $\xi$ отображения

$$
f_{*}(\cdot, \xi): R^{*} \rightarrow R,
$$

где $R=F \cap N^{\perp}, f_{*}(u, \xi):=f\left(\sum_{j=1}^{n} \xi_{j} e_{j}+u\right)-\sum_{j=1}^{n}\left\langle e_{j}, f\left(\sum_{j=1}^{n} \xi_{j} e_{j}+u\right)\right\rangle$.

При выполнении этих условий уравнение

$$
f_{*}(u, \xi)=0
$$


однозначно разрешимо при всех $\xi$, и его решение $u=h(\xi)$ гладко зависит от $\xi$ - по теореме о неявной функции. Из (2.1.4) получаем

$$
W(\xi) \equiv V\left(\sum_{j=1}^{n} \xi_{j} e_{j}+h(\xi)\right)
$$

Переход от $V$ к $W$, определенной соотношением (2.1.5) (или (2.1.8)), аналогичен редукции Пуанкаре в конечномерном случае.

Первые реализации идеи конечномерной редукции были сделаны А. М. Ляпуновым и Э. Шмидтом [19], [20]. Правда, вместо функции (2.1.8) они рассматривали так называемое уравнение разветвления

$$
\theta(\xi)=0, \quad \xi \in \mathbb{R}^{n},
$$

где $\theta(\xi)=\left(\theta_{1}(\xi), \ldots, \theta_{n}(\xi)\right), \theta_{j}(\xi)=\left\langle f\left(\sum_{k=1}^{n} \xi_{k} e_{k}+h(\xi)\right), e_{j}\right\rangle .^{3}$.

Нетрудно заметить, что

$$
\theta(\xi)=\operatorname{grad} W(\xi)
$$

Подходы А. М. Ляпунова и Э. Шмидта получили дальнейшее развитие в работах многочисленных математиков, что впоследствии привело к созданию обширной теории ветвления решений нелинейных уравнений [21], [38]. В этой теории произошел отрыв от вариационных корней метода конечномерных редукций. Возврат к вариационным задачам в рамках обшей теории ветвления произошел лишь в 70-е годы [2], [3], [4].

Следует отметить, что развитие упомянутых подходов шло, в основном, на уровне локальных задач - в виде схем построения так называемых малых решений, зависящих от малых параметров. Потребность в условиях типа собственности отображения (2.1.6) при этом не возникала. Нелокальное рассмотрение ключевых функций было востребовано в связи с некоторыми новыми задачами, пришедшими из симплектической топологии [22]. В работах [22], [23] для построения нелокальных редукций вместо условия собственности отображения (2.1.6) использовались более частные условия, приводящие к возможности применения принципа сжатых отображений. Впервые условие собственности в проблеме редукции было использовано, по-видимому, в работе [39]. В этой работе было сформулировано следуюшее утверждение.

ТЕОРема 2.1.1. Пусть отображение (2.1.6) является собственным, и пусть при әтом выполняется условие положительности (2.1.4). Тогда маргинальное отобрахсение $\varphi: \xi \mapsto \sum_{j=1}^{n} \xi_{j} e_{j}+h(\xi)$, где $h(\xi)$ определено уравнением (2.1.7), устанавливает взаимно однозначное соответствие между критическими точками ключевой функции (2.1.5) и заданного функционала V. При этом локальныее

\footnotetext{
${ }^{3}$ Такое определение уравнения разветвления дал А.М. Ляпунов. Определение, данное Э. Шмидтом, выглядит несколько иначе, но приводит к эквивалентному уравнению [38].
} 
кольца особенностей ${ }^{4}$ соответствуюших функиий в точках $\xi$ и $\varphi(\xi)$ изоморфны. В соответствующих друг другу однократных критических точках имеет место совпадение индексов Морса ${ }^{5}$.

Доказательство изоморфности локальных колец проводится стандартными приемами, разработанными в конечномерной теории особенностей гладких функций [26]. Эти приемы легко переносятся на бесконечномерные пространства в случае фредгольмовых особенностей.

Доказательство остальных частей утверждения - дословное повторение соответствуюших пунктов доказательств теорем 1.1.1 и 1.1.2.

Следующий шаг в развитии данной схемы - замена линейных функционалов $p_{j}$, $p_{j}(x)=\left\langle x, e_{j}\right\rangle($ см. (2.1.5)) на более сложные линейные и нелинейные функционалы. Например, в двухточечной краевой задаче, отвечающей потенциалу $V(x)=$ $\int_{0}^{1}\left(\frac{\dot{x}^{2}}{2}+u(x)\right) d t$, можно, следуя Морсу и Ботту [7], ввести ключевую функцию $\widetilde{W}$,

$$
\widetilde{W}(\xi):=\inf _{x: \widetilde{p}_{j}(x)=\xi_{j}, j=1, \ldots, n} V(x)
$$

где $\widetilde{p}_{j}(x):=x(j /(n+1))$. Функция $\widetilde{W}$ стабильно гладко эквивалентна функции (2.1.5), определенной посредством системы векторов $\left\{e_{j}(t)=\sqrt{2} \sin (\pi j s)\right\}_{j=1}^{n}$ (функции называются стабильно эквивалентными, если они становятся эквивалентными после добавления к ним невырожденных квадратичных форм дополнительных переменных [26]). Более подробные сведения о редукциях в данной краевой задаче имеются в $\S 2.4$ (см. также [39], [40]).

В следуюшем параграфе будет рассматриваться редуцирующая схема Морса-Ботта в ее естественном окружении.

ЗАмЕЧАнИЕ 2.1.1. Условие собственности отображения (2.1.6) можно заменить на любое другое условие, гарантирующее существование условных экстремалей в слоях $p^{-1}(\xi), p(x)=\left(p_{1}(x), \ldots, p_{n}(x)\right)^{T}$. Например, если пространство $E$ рефлексивно, то достаточно потребовать коэрцитивности $V$ (наряду с вьпуклостью) вдоль каждого слоя.

В этих же целях можно применять и известное условие (С) Пале-Смейла [41].

ЗАмЕчАниЕ 2.1.2. Если на $H$ зафиксировано ортогональное действие некоторой группы $G$ с условием инвариантности подпространств, $F, E, N=\operatorname{span}\left(e_{1}, \ldots, e_{n}\right)$ и функционала $V$ относительно этого действия, то ключевая функция (2.1.5) будет инвариантной относительно действия $G$ на $N$.

\footnotetext{
${ }^{4}$ Локальное кольцо особенности гладкого функционала $V$ в критической точке $a$ определяется как фактор кольца ростков гладких функционалов в точке $a$ по идеалу, порожденному функционалами вида $\alpha(f(x))$, где $\alpha$ - произвольный гладкий функционал, заданный на произвольной окрестности нуля в пространстве $F\left(f=\operatorname{grad}_{H} V\right)$.

${ }^{5}$ Индекс Морса в бесконечномерном случае определяется как максимальная размерность подпространства, на котором отрицательно определен второй дифференциал.
} 


\section{§ 2.2. Редукция Морса-Ботта}

Пусть $\mathscr{M}$ - компактное связное риманово $C^{\infty}$-многообразие без края и $a, b-$ фиксированная пара точек на $\mathscr{M}$. Через $\mathscr{X}$ обозначим множество $H^{1}$-кривых $x:[0,1] \rightarrow \mathscr{M}$, соединяюших $a$ и $b(x(0)=a, x(1)=b)$. Принадлежность кривой $x=x(t)$ классу $H^{1}$ означает ее абсолютную непрерывность и суммируемость скалярного квадрата скорости $|\dot{x}(t)|^{2}=(\dot{x}(t), \dot{x}(t))_{x(t)}$. Через $(\cdot, \cdot)_{a}$ обозначается скалярное произведение в касательном пространстве $T_{a}(\mathscr{M}), a \in \mathscr{M}$. В $\mathscr{X}$ естественно вводится бесконечномерная риманова структура [41] с модельным гильбертовым пространством

$$
X=\left\{u(t) \in H^{1}\left([0,1], \mathbb{R}^{m}\right): u(0)=u(1)=0\right\}, \quad m=\operatorname{dim} \mathscr{M} .
$$

Функционал действия $V(x)=\int_{0}^{1} \frac{\dot{x}^{2}}{2} d t$ на $\mathscr{X}$ является гладким и достигает своего минимального значения на кратчайшей кривой $\gamma$, соединяюшей $a$ и $b[6],[7],[41]$. Пусть $c$ - регулярное значение $V, V(\gamma)>c$. Нетрудно установить существование такой величины $\varepsilon>0$, что для любой кривой $x=x(t)$ в $\{V \leqslant c\}$ и любой пары точек $t, s \in[0,1]$, для которой $|t-s|<\varepsilon$, точки $x(t)$ и $x(s)$ принадлежат геодезически вьпуклому открытому подмножеству в $\mathscr{M}$. Пусть целое положительное число $n$ таково, что $1 /(n+1)<\varepsilon$. Рассмотрим $\mathscr{M}^{n}=\underbrace{\mathscr{M} \times \cdots \times \mathscr{M}}_{n}$ и подмножество $\mathscr{N}_{c} \subset \mathscr{M}^{n}$, состоящее из таких точек $\left(q_{1}, \ldots, q_{n}\right)$, для которых

$$
\sum_{j=0}^{n} \int_{j /(n+1)}^{(j+1) /(n+1)}\left|\dot{\gamma}_{j, q}\right|^{2} d t \leqslant 2 c
$$

$\gamma_{j, q}-$ кратчайшая параметрическая кривая с параметром $t \in[j /(n+1),(j+1) /(n+1)]$, соединяющая $q_{j}$ и $q_{j+1}\left(q_{0}=a, q_{n+1}=b\right)$.

Легко увидеть, что отображения

$$
\begin{gathered}
p:\{V \leqslant c\} \rightarrow \mathscr{N}_{c}, \quad \varphi: \mathscr{N}_{c} \rightarrow\{V \leqslant c\}, \\
p(x)=(x(1 /(n+1)), \ldots, x(n /(n+1)))^{T}, \\
\varphi(q)(t):=\gamma_{j, q}(t) \quad \forall t \in[j /(n+1),(j+1) /(n+1)],
\end{gathered}
$$

являются гладкими ${ }^{6}$. При этом $p(\varphi(q)) \equiv q$ и $V(\varphi(q))=\inf _{x: p(x)=q} V(x)$.

Нетрудно проверить, что $\varphi(q)$ - невырожденная точка минимума для $V$.

Отображения $p$ и $\varphi$ гомотопически обратны друг другу. Процедура стягивания слоев $p^{-1}(q)$ в точки $\varphi(q)$ детально описана в [7].

ЗАмЕчАнИЕ 2.2.1. Если на $\mathscr{M}$ задано действие группы $G$ с условиями $g(a)=a$, $g(b)=b \forall g \in G$ и инвариантностью функционала действия относительно преобразований

$$
x(t) \mapsto g(x(t)) \quad \forall g \in G,
$$

то ключевая функция $W, W(q):=V(\varphi(q))$, будет инвариантной относительно преобразований $\left(q_{1}, \ldots, q_{n}\right) \rightarrow\left(g\left(q_{1}\right), \ldots, g\left(q_{n}\right)\right)$.

\footnotetext{
${ }^{6}$ Гладкость отображения в точке края означает существование локального гладкого продолжения отображения за край (на открытую окрестность заданной точки края в объемлющем многообразии).
} 


\section{§ 2.3. Общая схема конечномерной редукции}

Редукции Ляпунова-Шмидта и Морса-Ботта можно включить как частные случаи в общую схему.

ОПРЕДЕЛЕниЕ 2.3.1. Пусть $V$ - гладкий функционал, заданный на гладком банаховом многообразии $\mathscr{X}$. Конечномерной редукиией $V$ на открытом подмножестве $\mathscr{O} \subset \mathscr{X}$ назьвается тройка $\{p, \varphi, \mathscr{N}\}$, в которой $\mathscr{N}$ - гладкое конечномерное многообразие, $p$ - гладкая субмерсия из $\mathscr{O}$ на $\mathscr{N}, \varphi$ - гладкое отображение из $\mathscr{N}$ в $\mathscr{O}$, секушее $p(p \circ \varphi=I)$, при условии выполнения следуюших двух требований:

1) $\varphi(\xi)$ - единственная критическая точка сужения $\left.V\right|_{p^{-1}(\xi)} \forall \xi \in \mathscr{N}$;

2) $\varphi(\xi)$ - невырожденная критическая точка $\left.V\right|_{p^{-1}(\xi)} \forall \xi \in \mathscr{N}$ (невырожденность критической точки означает невырожденность второго дифференциала

$$
\frac{\partial^{2}}{\partial x^{2}}\left(\left.V\right|_{p^{-1}(\xi)}\right)(\varphi(\xi))(h, h), \quad h \in T_{\varphi(\xi)}\left(p^{-1}(\xi)\right)
$$

как квадратичной формы на линейном пространстве).

Если к условиям определения 2.3.1 добавлено требование " $\varphi(\xi)$ - точка глобального минимума $\left.V\right|_{p^{-1}(\xi)} \forall \xi \in \mathscr{N}$ ", то редукция назьвается әллиптической.

Как и ранее, функция $W$

$$
W(\xi):=\inf _{x: p(x)=\xi} V(x)=V(\varphi(\xi))
$$

назьвается ключевой.

TеОРема 2.3.1. Пусть $\{p, \varphi, \mathscr{N}\}$ - әллиптическая конечномерная редукиия гладкого функиионала $V$ на области $\mathscr{O}$ гладкого банахова многообразия $\mathscr{X}$.

Тогда отображение (маргинальное) ч устанавливает взаимно однозначное соответствие между множествами критических точек маргинальной функиии (2.3.1) и функиионала V. При этом соответствующие друг другу критические точки одновременно являются либо вырожденными, либо нет. В случае невырожденности соответствующие критические точки имеют одинаковые значения индексов Морса.

ЗАмЕчАниЕ 2.3.1. Снятие требования эллиптичности в формулировке теоремы 2.3.1 приводит к снятию утверждения о совпадении значений индексов Морса.

ДОКАЗАТЕЛЬСТВО ТЕОРЕМЫ. ИЗ условия теоремЫ следует, что подмножество $\mathscr{L}=\{\varphi(\xi)\}_{\xi \in \mathcal{N}}$ является гладким подмногообразием в $\mathscr{X}$. Если $\bar{x}=\varphi(\xi)$, то $T_{\bar{x}}(\mathscr{X})=T_{\bar{x}}(\mathscr{L})+T_{\bar{x}}\left(p^{-1}(\xi)\right)$. Пусть $h=h_{1}+h_{2}, h_{1} \in T_{\bar{x}}(\mathscr{L}), h_{2} \in T_{\bar{x}}\left(p^{-1}(\bar{\xi})\right)$. Так как $\bar{x}$ - критическая точка для $\left.V\right|_{p^{-1}(\bar{\xi})}$, то $\frac{\partial V}{\partial x}(\bar{x}) h_{2}=0$ и $\frac{\partial V}{\partial x}(\bar{x}) h=\frac{\partial V}{\partial x}(\bar{x}) h_{1}$. Из равенства $W(\xi)=V(\varphi(\xi))$ получаем $\frac{\partial W}{\partial \xi}(\xi) \eta=\frac{\partial V}{\partial x}(\bar{x}) h_{1}$, где $\eta$ - такой вектор в $T_{\bar{\xi}}(\mathscr{N})$, для которого $h_{1}=\frac{\partial \varphi}{\partial \xi}(\bar{\xi}) \eta$. Следовательно, равенство нулю дифференциала 
$\frac{\partial V}{\partial x}(\bar{x})$ равносильно равенству нулю дифференциала $\frac{\partial W}{\partial \xi}(\xi)$, что и доказывает первое утверждение теоремы.

Для доказательства остальных утверждений положим $\mathscr{N}=\mathbb{R}^{n}$ и $\xi=\left(\xi_{1}, \ldots, \xi_{n}\right)^{T}$ (в силу локальности утверждений это предположение не ограничивает обшности рассуждения). Далее, заметим, что из равенства $W(\xi)=V(\varphi(\xi))$ вытекает

$$
\frac{\partial^{2} W}{\partial \xi_{i} \partial \xi_{j}}(\bar{\xi})=\frac{\partial^{2} V}{\partial x^{2}}(\bar{x})\left(\frac{\partial \varphi}{\partial \xi_{i}}(\bar{\xi}), \frac{\partial \varphi}{\partial \xi_{j}}(\bar{\xi})\right)
$$

а из тождества

$$
\left.\frac{\partial V}{\partial x}(\varphi(\xi))\right|_{T_{\varphi(\xi)}\left(p^{-1}(\xi)\right)}=0
$$

следует

$$
\frac{\partial^{2} V}{\partial x^{2}}(\bar{x})\left(h_{1}, h_{2}\right)=0, \quad h_{1} \in T_{\bar{x}}(\mathscr{L}), \quad h_{2} \in T_{\bar{x}}\left(p^{-1}(\bar{\xi})\right) .
$$

Но так как, по условию, квадратичная форма

$$
\frac{\partial^{2} V}{\partial x^{2}}(\bar{x})\left(h_{2}, h_{2}\right)=\frac{\partial^{2}}{\partial x^{2}}\left(\left.V\right|_{p^{-1}(\bar{\xi})}(\bar{x})\right)\left(h_{2}, h_{2}\right)
$$

является невырожденной, то невырожденность формы

$$
\left.\frac{\partial^{2} V}{\partial x^{2}}(\bar{x})(h, h)=\frac{\partial^{2} V}{\partial x^{2}}(\bar{x})\left(h_{1}, h_{1}\right)+\frac{\partial^{2} V}{\partial x^{2}}(\bar{x})\right)\left(h_{2}, h_{2}\right)
$$

равносильна невырожденности первого слагаемого в правой части (2.3.2). И так как

$$
\frac{\partial^{2} W}{\partial \xi^{2}}(\bar{\xi})(\eta, \eta)=\frac{\partial^{2} V}{\partial x^{2}}(\bar{x})\left(h_{1}, h_{1}\right), \quad h_{1}=\sum \eta_{j} \frac{\partial \varphi}{\partial \xi_{j}}(\bar{\xi}), \quad \eta=\left(\eta_{1}, \ldots, \eta_{n}\right)^{T},
$$

то невырожденность этого слагаемого равносильна невырожденности $W$ в критической точке $\bar{\xi}$. Второе утверждение доказано.

При вьполнении требования эллиптичности форма $\frac{\partial^{2} V}{\partial x^{2}}(\bar{x})\left(h_{2}, h_{2}\right)$ будет положительной, и, следовательно, индекс Морса левой части (2.3.2) совпадает с индексом Морса формы $\frac{\partial^{2} W}{\partial \xi^{2}}(\bar{\xi})(\eta, \eta)$. Теорема полностью доказана.

ОПРЕДЕЛЕнИЕ 2.3.2. Редукция $\{\widetilde{p}, \widetilde{\varphi}, \widetilde{N}\}$ функционала $V$ на области $\mathscr{O} \subset \mathscr{X}$ назьвается расширением редукции $\{p, \varphi, \mathscr{N}\}$ этого же функционала $V$ на области $\mathscr{O}$ (соответственно, $\{p, \varphi, \mathcal{N}\}$ называется сужением редукции $\{\widetilde{p}, \widetilde{\varphi}, \widetilde{\mathcal{N}}\})$, если сушествует такая редукция $\{\pi, \psi, \mathcal{N}\}$ для $\widetilde{W}$ на $\widetilde{\mathcal{N}}$, что $p=\pi \circ \widetilde{p}$ и $\varphi=\widetilde{\varphi} \circ \psi$.

ЗАмЕчАНИЕ 2.3.2. В условиях определения 2.3.2 имеет место соотношение $W(\xi)=$ $\widetilde{W}(\psi(\xi))$, где $W(\xi)=V(\varphi(\xi))$ и $\widetilde{W}(\widetilde{\xi})=V(\widetilde{\varphi}(\widetilde{\xi}))$.

$\mathrm{B}$ случае эллиптичности всех редукций получаем $W(\xi)=\inf _{\widetilde{\xi}: \pi(\widetilde{\xi})=\xi} \widetilde{W}(\widetilde{\xi})$. 
Tеорема 2.3.2. Пусть пара редукиий $\{p, \varphi, \mathscr{N}\}$ u $\{\widetilde{p}, \widetilde{\varphi}, \widetilde{N}\}$ функционала $V$ на области $\mathscr{O} \subset \mathscr{X}$ имеют общее расширение (то есть обе редукиии являются сужениями третьей редукиии). Тогда локальные кольиа особенностей функиий $W$ и $\widetilde{W}$ в критических точках $a \in \mathcal{N}$ u $\widetilde{a} \in \widetilde{\mathscr{N}}$, в которых $\varphi(a)=\widetilde{\varphi}(\widetilde{a})$, изоморфны.

Доказательство достаточно провести лишш в случае, в котором $\{p, \varphi, \mathscr{N}\}$ - сужение $\{\widetilde{p}, \widetilde{\varphi}, \widetilde{N}\}$. Но в этом случае данное утверждение является следствием теоремы 1.1.2 (см. замечание 2.3.2).

ЗАмечАниЕ 2.3.3. Из результатов работы [42] следует, что при $\operatorname{dim} \mathscr{N}=\operatorname{dim} \widetilde{\mathscr{N}}$ и эллиптичности обеих редукций ростки функций $W$ и $\widetilde{W}$ в точках $a$ и $\widetilde{a}$, в которых $\varphi(a)=\widetilde{\varphi}(\widetilde{a})$, гладко эквивалентны. Создается впечатление, что $W$ и $\widetilde{W}$ должны быть и глобально гладко эквивалентными. Однако автор не располагает доказательством этого утверждения.

ЗАмечание 2.3.4. Пусть на $\mathscr{O} \subset \mathscr{X}$ и $\mathscr{N}$ зафиксированы действия $G \times \mathscr{O} \rightarrow \mathscr{O}$ и $G \times \mathscr{N} \rightarrow \mathcal{N}$ групшы $G$ с условиями инвариантности $V$ относительно действия $G$ на $\mathscr{O}$ и эквивариантности субмерсии относительно обоих действий (здесь $\{p, \varphi, \mathscr{N}\}$ - редукция для $V$ на $\mathscr{O})$. Тогда ключевая функция $W(\xi):=V(\varphi(\xi))$ инвариантна относительно заданного действия $G$ на $\mathcal{N}$.

ЗАмЕЧАнИЕ 2.3.5. Для установления условий существования редукций функционала $V$ на тех или иных областях многообразия $\mathscr{X}$ можно использовать дополнительные бесконечномерные римановы оснащения на $\mathscr{X}$. При этом могут, в отличие от конечномерного случая, появляться специфические трудности, связанные с отличием основной топологии на $\mathscr{X}$ от топологии, порожденной римановой метрикой.

Эти трудности иногда можно преодолеть, потребовав от функционала $V$ вьполнения дополнительных свойств типа фредгольмовости второго ковариантного дифференциала и т.п. Соответствующие точные определения и утверждения имеются в [40].

\section{§ 2.4. Упругие равновесия эйлерова стержня}

Упругое равновесие продольно сжатого прямолинейного плоского (эйлерова) стержня описьвается уравнением [13], [43]

$$
\ddot{x}+\lambda \sin x=0,
$$

в котором $\lambda$ - параметр силы продольного сжатия стержня, $x(s)$ - угловая функция (угол между касательной к упругой линии и вертикалью), $s$ - параметр длины стержня. Если предположить, что концы стержня жестко закреплены, то к уравнению (2.4.1) необходимо добавить краевое условие

$$
x(0)=x(1)=0
$$

(длина стержня предполагается единичной).

Пусть $E$ - пространство дважды непрерьвно дифференцируемых функций на [0,1], удовлетворяюших краевому условию (2.4.2). Отображение $f: E \rightarrow F, F=C([0,1])$, 
заданное левой частью уравнения (2.4.1), является фредгольмовым индекса нуль и потенциальным. Его потенциалом будет взятый с противоположным знаком функционал полной энергии $V$

$$
V(x, \lambda)=\int_{0}^{1}\left(\frac{\dot{x}^{2}}{2}+\lambda(\cos x-1)\right) d s
$$

Пусть $p: E \rightarrow \mathbb{R}^{n}$ - отображение, для которого

$$
p(x)=\left(p_{1}(x), \ldots, p_{n}(x)\right), \quad p_{j}(x)=\int_{0}^{1} e_{j}(s) x(s) d s, \quad e_{j}(s)=\sqrt{2} \sin (\pi j s) .
$$

Действуя в соответствии с редуцируюшей схемой Ляпунова-Шмидта, рассмотрим функцию $W$

$$
W(\xi, \lambda):=\inf _{x: p(x)=\xi} V(x, \lambda), \quad \xi \in \mathbb{R}^{n}
$$

Если $\lambda$ подчинено ограничению

$$
\lambda<\pi^{2}(n+1)^{2}
$$

то функция (2.4.4) будет ключевой. Действительно, для функции (2.4.4) имеет место представление

$$
W(\xi, \lambda)=V\left(\sum_{j=1}^{n} \xi_{j} e_{j}+\varphi(\xi, \lambda), \lambda\right),
$$

в котором вектор $u=\varphi(\xi, \lambda)$ ортогонален (в метрике $\left.L_{2}([0,1])\right)$ подпространству $N=$ $\operatorname{span}\left(e_{1}, \ldots, e_{n}\right)$ и является решением уравнения

$$
f_{*}(u, \xi, \lambda)=0, \quad u \in N^{\perp}
$$

где

$$
f_{*}(u, \xi, \lambda):=f\left(\sum_{j=1}^{n} \xi_{j} e_{j}+u, \lambda\right)-\sum_{k=1}^{n}\left\langle f\left(\sum_{j=1}^{n} \xi_{j} e_{j}+u, \lambda\right), e_{k}\right\rangle e_{k}
$$

$\left(\langle x, y\rangle:=\int_{0}^{1} x(s) y(s) d s\right)$. Отображение $f_{*}(\cdot, \xi, \lambda): E_{*} \rightarrow F_{*}$, где $E_{*}=E \cap N^{\perp}$, $F_{*}=F \cap N^{\perp}$, представимо в виде суммы линейного изоморфизма $u \mapsto \ddot{u}$ и отображения с ограниченным образом. Следовательно, оно является собственным.

Дифференциал отображения $f_{*}(\cdot, \xi, \lambda)$ в произвольной точке $u \in E_{*}$ задается соответствием

$$
h \mapsto \ddot{h}+\lambda(\cos \bar{x}) h-\lambda \sum_{j=1}^{n}\left(\int_{0}^{1}(\cos \bar{x}) h e_{j} d s\right) e_{j}
$$


где $\bar{x}=\sum \bar{\xi}_{j} e_{j}+u$. При ограничении (2.4.5) это соответствие задает положительный изоморфизм из $E_{*}$ на $F_{*}$. Данное утверждение является следствием равенства нулю фредгольмова индекса $f$, соотношения

$$
\frac{\partial^{2} V}{\partial x^{2}}(\bar{x})(h, h)=|\dot{h}|^{2}-\lambda\langle(\cos \bar{x}) h, h\rangle, \quad h \in E_{*},
$$

и оценок $|\dot{h}|^{2}>\pi^{2}(n+1)^{2}|h|^{2},|\langle(\cos x) h, h\rangle|<|h|^{2}$ (все скалярные произведения и нормы взяты из пространства $\left.H=L_{2}([0,1])\right)$. Следовательно, вьполнены все условия теоремы 2.1.1 и поэтому от (2.4.3) к (2.4.4) является редуцируюшим.

Таким образом, анализ равновесных конфигураций эйлерова стержня сводится к анализу поведения ключевой функции (2.4.4).

В этой же задаче можно воспользоваться редукцией Морса-Ботта, после применения которой (при ограничении (2.4.5)) анализ решений уравнения (2.4.1) сводится к анализу гладкой функции $\widetilde{W}$

$$
\widetilde{W}(\xi, \lambda):=\inf _{x: \tilde{p}(x)=\xi} V(x, \lambda), \quad \xi \in \mathbb{R}^{n},
$$

где $\widetilde{p}(x)=(x(1 /(n+1)), \ldots, x(n /(n+1)))$. Значения маргинального отображения в этой редуцирующей схеме "выходят" в более широкое пространство функций, допускаюших разрьвы производных в точках $1 /(n+1), 2 /(n+1), \ldots, n /(n+1)$. Сравнение обеих редукций можно провести, если их рассмотреть при $E=F=H=\stackrel{\circ}{W}_{2}^{1}$ $\left(\stackrel{\circ}{W} \underset{2}{1}\right.$ - пространство соболевских функций класса $W_{2}^{1}([0,1])$, удовлетворяющих краевому условию (2.4.3)). Обе редукции имеют общее расширение, полученное на основе объединения компонент редуцируюших отображений $p$ и $\widetilde{p}$. Таким образом, можно утверждать, что ключевые функции $W$ и $\widetilde{W}$ имеют одинаковые топологические и аналитические свойства (см. [39], [40]).

ЗАмЕчАниЕ 2.4.1. Так как уравнение (2.4.1) интегрируемо [43], то маргинальное отображение и ключевая функция допускают в данном случае явное представление через эллиптические функции Якоби и интегралы от них. Соответствующие точные вычисления проведены О. Н. Левченко [49], [50] (см. § 2.5).

ЗАмечАниЕ 2.4.2. При переходе $\lambda$ через первое критическое значение $\lambda=\pi^{2}$ (критическую нагрузку Эйлера) происходит потеря устойчивости стержня в его нулевом (прямолинейном) состоянии. Анализ возникающей при этом бифуркации устойчивых ненулевых состояний сводится к изучению локального поведения функции $W$

$$
W(\xi, \lambda):=\inf _{x:\left\langle x, e_{1}\right\rangle=\xi} V(x, \lambda), \quad \xi \in \mathbb{R} .
$$

Поведение $W(\xi, \lambda)$ (при $(\xi, \lambda)$, близких к точке $\left.\left(0, \pi^{2}\right)\right)$ в основном определяется главной частью этой функции, имеюшей вид $-(\delta / 2) \xi^{2}+a \xi^{4}, a>0, \delta=\lambda-\lambda_{1}$. Точно такую же главную часть имеет и ритцевская аппроксимация потенциала $W_{R}, W_{R}(\xi, \lambda)$ $:=V\left(\xi e_{1}, \lambda\right)$. 


\section{§ 2.5. Редукция Морса-Ботта для кирхгофова стержня}

Задача о формах равновесия пространственного упругого стержня в модели Кирхгофа [43], [44] исследовалась многими на основе кинетической аналогии с задачей о движении твердого тела вокруг неподвижной точки [43]-[45]. При этом детально были проанализированы ситуации, отвечающие случаям полной интегрируемости уравнений равновесия. В [46]-[48] были исследованы бифуркации закритических равновесий кирхгофова стержня локальным методом Ляпунова-Шмидта без предположения интегрируемости.

Применение метода Ляпунова-Шмидта требует предварительного введения системы координат на окрестности единицы в $\mathrm{SO}(3)$. Для исследования поведения кирхгофова стержня в целом, по-видимому, целесообразнее использовать метод Морса-Ботта [49].

Равновесные конфигурации прямолинейного и продольно сжатого кирхгофова стержня длины единица с жестким закреплением концов описьваются краевой задачей

$$
\left\{\begin{array}{l}
A \dot{\omega}+[A \omega, \omega]+\lambda\left[r_{3}, f^{-1} r_{3}\right]=0 \\
f(0)=f(1)=I
\end{array}\right.
$$

Здесь $\lambda$ - параметр сжимающей нагрузки, $A=\operatorname{diag}\left(A_{1}, A_{2}, A_{3}\right)$ - тензор упругости в поперечном сечении $\left(A_{j}>0\right)$, для которого вьполняется условие Е. Л. Николаи: $A_{3}^{-1}>\frac{1}{2}(1+\nu)\left(A_{1}^{-1}+A_{2}^{-1}\right)(\nu-$ коэффициент Пуассона), $\omega(s)-$ угловая скорость движения нормального сечения стержня в зависимости от параметра длины $s$ средней линии стержня, записанная в координатах тройки ортов $f_{1}(s), f_{2}(s), f_{3}(s)$, направленных по осям инерции нормального сечения, $r_{3}=f_{3}(0)$. Орт $f_{3}(s)$ является касательным вектором к средней линии стержня, $f(s)$ - матричная функция, столбцами которой являются векторы $f_{1}(s), f_{2}(s)$ и $f_{3}(s) \quad\left(f_{j}(s)=f(s) r_{j}\right)$.

Уравнение (2.5.1) является уравнением Эйлера-Лагранжа экстремалей функционала полной энергии $V$

$$
V(f, \lambda):=\frac{1}{2}\langle A \omega, \omega\rangle+\lambda\left\langle r_{3}, f r_{3}\right\rangle
$$

$\left(\right.$ здесь $\left.\langle\varphi, \psi\rangle=\int_{0}^{1}(\varphi(s), \psi(s)) d s\right)$.

Вектор $\omega=\omega_{1} r_{1}+\omega_{2} r_{2}+\omega_{3} r_{3}$ канонически отождествляется [27] с матрицей

$$
\Omega=\left(\begin{array}{ccc}
0 & -\omega_{3} & \omega_{2} \\
\omega_{3} & 0 & -\omega_{1} \\
-\omega_{2} & \omega_{1} & 0
\end{array}\right)
$$

для которой имеет место представление

$$
\Omega(s)=f^{-1}(s) \frac{d f}{d s}(s)
$$


Пусть $\mathscr{M}$ - банахова группа Ли $C^{2}$-петель на $\mathrm{SO}(3)$ в единице:

$$
\mathscr{M}=\left\{f \in C^{2}([0,1], \mathrm{SO}(3)): f(0)=f(1)=I\right\} .
$$

Отображение $p: f(s) \mapsto \tau(s):=f(s) r_{3}$ задает гладкую субмерсию из $\mathscr{M}$ на гладкое банахово многообразие $\widetilde{\mathscr{M}}$ петель класса $C^{2}$ на двумерной сфере $S^{2}$ :

$$
\widetilde{\mathscr{M}}=\left\{\tau \in C^{2}\left([0,1], S^{2}\right): \tau(0)=\tau(1)=r_{3}\right\} .
$$

Прообраз $p^{-1}(\tau)$ любой петли $\tau \in \widetilde{\mathscr{M}}$ является орбитой правого действия

$$
G \times \mathscr{M} \rightarrow \mathscr{M}, \quad(g(s), f(s)) \mapsto f(s) g(s),
$$

банаховой группы Ли $G=\left\{g: g(s)=\exp \left(\varphi(s) R_{3}\right)\right\}$. Через $R_{3}$ обозначается представление вида (2.5.3) для вектора $r_{3}$. Функция $\varphi(s)$ принадлежит классу $C^{2}$ и для нее выполняется условие

$$
\varphi(0)=0, \quad \varphi(1)=2 \pi k, \quad k \in \mathbb{Z} .
$$

Следовательно, $p^{-1}(\tau)$ состоит из счетного набора компонент связности $\mathscr{N}_{k}(\tau)$, определяемых выбором $k$ в (2.5.4).

Теорема 2.5.1. В случае симметричного стержня $\left(A_{1}=A_{2}\right)$ имеет место представление

$$
V(f g, \lambda)=\int_{0}^{1}\left(A_{1} \frac{|\dot{\tau}|^{2}}{2}+A_{3} \frac{\left(\omega_{3}+\dot{\varphi}\right)^{2}}{2}\right) d s+\lambda\left\langle\tau, r_{3}\right\rangle
$$

где $g(s)=\exp \left(\varphi(s) R_{3}\right), \omega_{3}-$ третья компонента угловой скорости, $|\dot{\tau}(s)|-\kappa р и$ визна средней линии стержня.

ДокАЗАТЕЛЬСТво следует из соотношения $|\dot{\tau}(s)|^{2}=\omega_{1}^{2}(s)+\omega_{2}^{2}(s)$, вытекаюшего из равенства

$$
\tau(s)=f(s) r_{3}, \quad\left|\dot{f}(s) r_{3}\right|=\left|f^{-1}(s) \dot{f}(s) r_{3}\right|=\left|\Omega r_{3}\right|=\left|\left[\omega, r_{3}\right]\right|
$$

и соотношения

$$
(f g)^{-1} \frac{d}{d s}(f g)=g^{-1} f^{-1}(\dot{f} g+f \dot{g})=g^{-1}\left(\Omega+\dot{\varphi} R_{3}\right) g .
$$

Из представления (2.5.5) получаем, что $\left.V\right|_{p^{-1}(\tau)}$ имеет ровно по одной точке минимума на каждой компоненте $\mathscr{N}_{k}(\tau)$. Причем $f \in \mathscr{N}_{k}(\tau)$ является точкой минимума $\left.V\right|_{p^{-1}(\tau)}$ тогда и только тогда, когда выполняется равенство

$$
\int_{0}^{1} \omega_{3}^{2} d s=c_{k}^{2}
$$

где $c_{k}=2 \pi k+\int_{0}^{1} \omega_{3} d s$ (интеграл $\int_{0}^{1} \omega_{3} d s$ является инвариантом правого действия $G$ на $\mathscr{M})$. Нетрудно установить, что ограничение $\left.V\right|_{p^{-1}(\tau)}$ является геодезически выпукльм в бесконечномерной римановой метрике, в которой расстояние между $f(s)$ и $f(s) g(s), g(s)=\exp \left(\varphi(s) R_{3}\right)$, измеряется интегралом $\int_{0}^{1} \varphi^{2}(s) d s$. Следовательно,
имеет место следующее утверждение. 
Теорема 2.5.2. Пусть $\mathscr{O}=\left\{f \in \mathscr{M}:\left|\int_{0}^{1} \omega_{3} d s\right|<\pi\right\}$. Тогда функиионал $\widetilde{V}$

$$
\widetilde{V}(\tau, \lambda):=\inf _{f: f \in \mathscr{O} \cap p^{-1}(\tau)} V(f, \lambda), \quad \tau \in p(\mathscr{O})
$$

является гладким и для него имеет место представление

$$
\widetilde{V}(\tau, \lambda)=\int_{0}^{1}\left(A_{1} \frac{|\dot{\tau}|^{2}}{2}+\lambda\left(\tau, r_{3}\right)\right) d s+\frac{1}{2}\left(\int_{0}^{1} \omega_{3} d s\right)^{2} .
$$

Данная теорема означает возможность редукции (бесконечномерной), позволяющей сводить изучение поведения $V$ на области $\mathscr{O}$ к изучению $\widetilde{V}$ на области $p(\mathscr{O}) \subset \widetilde{\mathscr{M}}$. Из геодезической выпуклости $V$ на $p^{-1}(\tau)$ следует, что критические точки $V$ на $\mathscr{O}$ взаимно однозначно соответствуют критическим точкам $\widetilde{V}$ на $p(\mathscr{O})$. При этом невырожденные критические точки переходят в невырожденные и только невырожденные с сохранением значений индекса Морса, а соответствующие друг другу вырожденњые критические точки имеют изоморфные локальные кольца особенностей.

Если $\lambda<4 \pi^{2}$, то дальнейший анализ функционала (2.5.7) можно осуществить через конечномерную редукцию Морса-Ботта к ключевой функции $W$

$$
W(\xi, \lambda):=\inf _{\tau: \tau(1 / 2)=\xi} \tilde{V}(\tau, \lambda), \quad \xi \in S^{2} .
$$

ТЕОрема 2.5.3. Если $\lambda<4 \pi^{2}$, то функиия (2.5.8) является гладкой на $S^{2} \backslash\left\{-r_{3}\right\}$. При әтом ее критические точки взаимно однозначно соответствуют критическим точкам $\widetilde{V}$ на $p(\mathscr{O})$ с сохранением значений индексов Морса и типов локальных колец, особенностей.

Доказательство вытекает из теорем 2.3.1, 2.3.2 и 2.1.1 (см. [40]).

Функцию (2.5.8) можно получить в явном виде [49], [50]. Действительно, маргинальное отображение $\xi \mapsto \tau_{\xi}$ здесь допускает представление в виде

$$
\tau_{\xi}(s)=(\cos \varphi(s)) r_{3}+(\sin \varphi(s)) r, \quad r \perp r_{3},
$$

где $\varphi(s)$ получено склейкой решений $\varphi_{1}(s)$ и $\varphi_{2}(s)$ уравнения

$$
\ddot{\varphi}+\lambda \sin \varphi=0
$$

отвечающих краевым условиям $\varphi_{1}(0)=0, \varphi_{1}(1 / 2)=\psi$ и $\varphi_{2}(1 / 2)=\psi, \varphi_{2}(1)=0$, $\psi=\arccos \left(r_{3}, \xi\right)$. Произведя в уравнении (2.5.9) стандартную подстановку [13], [43]

$$
\varphi_{1}=2 \arcsin (k \sin u)
$$

где $k$ - константа, $0<k<1$, получим, что $u$ является решением уравнения $\dot{u}=$ $\lambda^{1 / 2}\left(1-k^{2} \sin ^{2} u\right)^{1 / 2}$. Следовательно,

$$
u(s)=\operatorname{am}(\sqrt{\lambda} s ; k),
$$


где $\operatorname{am}(\sqrt{\lambda} s ; k)$ - так называемая амплитуда, полученная обращением эллиптического интеграла первого рода (в нормальной тригонометрической форме Лежандра):

$$
\sqrt{\lambda} s=F(u ; k):=\int_{0}^{u} \frac{d y}{\left(1-k^{2} \sin ^{2} y\right)^{1 / 2}} .
$$

Из (2.5.10) и (2.5.11) получаем явную формулу решения

$$
\varphi_{1}(s)=2 \arcsin (k \operatorname{sn}(\sqrt{\lambda} s ; k)),
$$

где $\operatorname{sn}(\tau ; k):=\sin \operatorname{am}(\tau ; k)-$ эллиптический синус, $k=\sin (\psi / 2) / \operatorname{sn}(\sqrt{\lambda} / 2 ; k)$.

Для производной функции $\varphi_{1}$ получаем представление

$$
\dot{\varphi}_{1}(s)=2 \sqrt{\lambda} \operatorname{cn}(\sqrt{\lambda} s ; k),
$$

где $\operatorname{cn}(\tau ; k)=\cos \operatorname{am}(\tau ; k)$ - эллиптический косинус. Соответственно, для лагранжиана $L=\frac{\dot{\varphi}^{2}}{2}-\lambda(\cos \varphi-1)$ на решении $\varphi=\varphi_{1}(s)$ имеем представление

$$
L=2 \lambda k^{2}\left(\mathrm{cn}^{2}(\sqrt{\lambda} s ; k)-\operatorname{sn}^{2}(\sqrt{\lambda} s ; k)\right)=2 \lambda k^{2}\left(2 \mathrm{cn}^{2}(\sqrt{\lambda} s ; k)-1\right) .
$$

Решение $\varphi=\varphi_{2}(s)$ уравнения (2.5.10) получается продолжением $\varphi_{1}(s)$ с интервала $[0,1 / 2]$ на интервал $[1 / 2,1]$ по симметрии: $\varphi_{2}(s)=\varphi_{1}(1-s)$. Следовательно, $\int_{0}^{1} L d s=2 \int_{0}^{1 / 2} L d s$, и для ключевой функции (2.5.8) получаем представление

$$
W(\xi, \lambda)=4 \lambda k^{2}\left(2 \int_{0}^{1 / 2} \mathrm{cn}^{2}(\sqrt{\lambda} s ; k) d s-\frac{1}{2}\right)
$$

\section{§ 2.6. Равновесия тонкой упругой пластины}

Равновесное состояние упругой прямолинейной пластины, продольно сжатой и шарнирно закрепленной на крае, описьвается уравнениями Кармана (промасштабированными) [51], [52]:

$$
\Delta^{2} w-[w, \varphi]+\lambda w_{x x}=\Delta^{2} \varphi+\frac{1}{2}[w, w]=0
$$

при краевых условиях

$$
w=\Delta w=\varphi=\Delta \varphi=\left.0\right|_{\partial \Omega_{a}} .
$$

Через $w$ и $\varphi$ в этих уравнениях обозначены функции прогиба и напряжения пластины, $\Omega_{a}=[0, a] \times[0,1], \Delta-$ гармонический оператор Лапласа, $[w, \varphi]=$ $w_{x x} \varphi_{y y}+w_{y y} \varphi_{x x}-2 w_{x y} \varphi_{x y}, \lambda-$ параметр нагрузки. 
Пусть $H^{4}$ - пространство соболевских функций класса $W_{2}^{4}$, удовлетворяюших (2.6.2). Уравнение (2.6.1) является уравнением Эйлера-Лагранжа экстремалей функционала $V$

$$
V(w, \varphi, \lambda)=\frac{1}{2}\left(|\Delta w|^{2}-\lambda\left|w_{x}\right|^{2}-|\Delta \varphi|^{2}-\langle[w, w], \varphi\rangle\right)
$$

на пространстве $H^{4} \times H^{4}$. Здесь $|\varphi|^{2}=\langle\varphi, \varphi\rangle:=\iint_{\Omega_{a}} \varphi^{2}(x, y) d x d y$. Как правило, исследование функционала (2.6.3) проводится через редукцию к функционалу $\widehat{V}$

$$
\widehat{V}(w, \lambda):=\sup _{\varphi: \varphi \in H^{4}} V(w, \varphi, \lambda)
$$

Маргинальное отображение $H^{4} \rightarrow H^{4} \times H^{4}$, отвечающее данной редукции, задается соответствием

$$
w \mapsto\left(w,-\frac{1}{2} \Delta^{-2}[w, w]\right) .
$$

Следовательно, для (2.6.4) имеет место представление

$$
\widehat{V}(w, \lambda)=\frac{1}{2}\left(|\Delta w|^{2}-\lambda\left|w_{x}\right|^{2}\right)+\frac{1}{8}\left|\Delta^{-1}[w, w]\right|^{2} .
$$

Через $\Delta^{-1}$ обозначается оператор Грина $\psi \rightarrow \varphi$, где $\varphi-$ решение уравнения Пуассона $\Delta \varphi=\psi,\left.\varphi\right|_{\partial \Omega_{a}}=0$ (см. [53]). В [53] установлено, что оператор $\Delta$ действует как изоморфизм из пространства функций $\varphi$ гельдеровского класса $C^{2+\alpha}\left(\Omega_{a}\right)$, удовлетворяюших условию $\left.\varphi\right|_{\partial \Omega_{a}}=0$, на $F_{0}$ - подпространство функций $\psi$ в пространстве $F=C^{0+\alpha}\left(\Omega_{a}\right)$, удовлетворяющих условию согласования:

$$
\psi(0,0)=\psi(a, 0)=\psi(0,1)=\psi(a, 1)=0 .
$$

Обозначим теперь через $E$ банахово пространство $\left\{w \in H^{4}: \Delta^{2} w \in F\right\}$ с нормой $\|w\|_{E}:=\left\|\Delta^{2} w\right\|_{C^{0+\alpha}\left(\Omega_{a}\right)}$.

Через функционал (2.6.5) в теории упругих оболочек оценивается устойчивость равновесных состояний: состояние устойчиво, если оно реализует точку локального минимума функционала (2.6.5). Потеря устойчивости прямолинейной формы равновесия $(w=0)$ происходит при переходе $\lambda$ через критическое значение (верхнюю критическую нагрузку):

$$
\lambda^{*}(a)=\sup \left\{\bar{\lambda}: \frac{\partial^{2} \widehat{V}}{\partial w^{2}}(0, \lambda)(h, h)>0, \lambda<\bar{\lambda}, h \neq 0\right\} .
$$

Верхняя критическая нагрузка непрерьвно и кусочно гладко зависит от длины $a$ пластины. Разрыв производной $\lambda^{*}(a)$ происходит в точках $a_{m}=\sqrt{m(m+1)}, m=$ $1,2, \ldots$ При $a=a_{m}$ и $\lambda=\lambda_{m}:=\lambda^{*}\left(a_{m}\right)$ в нуле теряется устойчивость по двум модам:

$$
e_{1}=2 \sin \left(\frac{m \pi x}{a}\right) \sin (\pi y), \quad e_{2}=2 \sin \left(\frac{(m+1) \pi x}{a}\right) \sin (\pi y) .
$$


Анализ соответствующих закритических равновесий сводится к изучению критических точек ключевой функции $W$

$$
W(\xi, \lambda, a):=\inf _{u: u \in \mathscr{O}_{*}} V\left(\xi_{1} e_{1}+\xi_{2} e_{2}+u, \lambda\right), \quad \xi \in \mathscr{O}^{2}
$$

(при $(\lambda, a)$ достаточно близких к $\left.\left(\lambda_{m}, a_{m}\right)\right)$. Здесь $\mathscr{O}^{2}$ и $\mathscr{O}_{*}-$ достаточно малые окрестности нулей в $\mathbb{R}^{2}$ и $E_{*}=\left\{u \in E:\left\langle u, e_{1}\right\rangle=\left\langle u, e_{2}\right\rangle=0\right\}$. Локальный анализ (2.6.6) успешно осушествляется благодаря тому, что имеет место представление

$$
W(\xi, \lambda, a)=\frac{1}{2}\left(\alpha_{1}(\lambda, a) \xi_{1}^{2}+\alpha_{2}(\lambda, a) \xi_{2}^{2}\right)+\sum_{i, j=1}^{2} h_{i, j} \xi_{i}^{2} \xi_{j}^{2}+\cdots
$$

где $\alpha_{1}, \alpha_{2}$ - собственные значения оператора $\Delta^{2}+\lambda \frac{\partial^{2}}{\partial x^{2}}$, отвечаюшие собственным векторам $e_{1}, e_{2}$,

$$
\begin{gathered}
h_{j, j}=\frac{1}{8}\left|\Delta^{-1}\left[e_{j}, e_{j}\right]\right|^{2} \\
h_{1,2}=h_{2,1}=\frac{1}{8}\left(\left\langle\Delta^{-1}\left[e_{1}, e_{1}\right], \Delta^{-1}\left[e_{2}, e_{2}\right]\right\rangle+2\left|\Delta^{-1}\left[e_{1}, e_{2}\right]\right|^{2}\right) .
\end{gathered}
$$

Из результатов вычислений, опубликованных в [54], следует, что при нескольких начальных значениях $m$ выполняется неравенство

$$
h_{1,2}>\left(h_{1,1} h_{2,2}\right)^{1 / 2},
$$

благодаря которому можно осушествить полный локальный анализ бифуркации равновесий (см. [55], [56]).

ЗАМЕчАнИЕ 2.6.1. Неравенство (2.6.7) означает положительную определенность формы $\sum_{i, j=1}^{2} h_{i, j} \eta_{i} \eta_{j}$ в положительной четверти плоскости $\mathbb{R}^{2}$. Вопрос о знакоопределенности этой формы при произвольном $m$ пока остался открытым.

ЗАМЕчАНИЕ 2.6.2. Вопрос о существовании нелокальной редукции (гладкого продолжения ключевой функции (2.6.6) на конечную область при $\lambda>\lambda_{1}$ ) для прямоугольной пластины тоже пока открыт.

В аналогичной ситуации для круглой пластины вопрос глобальной конечномерной редуцируемости решен положительно [57]. 


\section{СПИСОК ЛИТЕРАТУРЫ}

[1] Обен ЖК.-П., Экланд И. Прикладной нелинейный анализ. М.: Мир, 1988.

[2] Треногин В. А., Сидоров Н. А. Исследование точек бифуркации и нетривиальных ветвей решений нелинейных уравнений // Дифференциальные и интегральные уравнения. № 1. Изд-во Иркутского ун-та, 1972. С. 216-247.

[3] Красносельский М. А., Бобылев Н.А., Мухамадиев Э. М. Об одной схеме исследования вырожденных экстремалей функционалов классического вариационного исчисления // ДАН СССР. 1978. Т. 240. № 3. С. 530-533.

[4] Marsden J. E. On the geometry of the Lyapunov-Schmidt procedure // Lect. Notes in Math. 1979. V. 755. P. 77-82.

[5] Заваровский Ю. Н. О методе Ляпунова-Шмидта для вариационных задач с параметром. Воронеж: ВГУ, 1981. Деп. в ВИНИТИ. № 478-82.

[6] Милнор Дж. Теория Морса. М.: Мир, 1965.

[7] Постников М. М. Введение в теорию Морса. М.: Наука, 1971.

[8] Chillingworth D. A global genericity theorem for bifurcations in variational problems // J. Funct. Anal. 1980. V. 35. P. 251-278.

[9] Magnus R. J. Universal unfolding in Banach spaces: reduction and stability // Mathematics Report 107. Geneva: Battele, 1977.

[10] Gromoll D., Meyer W. On differentiable functions with isolated critical points // Topology. 1969. V. 8. P. 361-370.

[11] Постон Т., Стюарт И. Теория катастроф и ее приложения. М.: Мир, 1980.

[12] Бобылев Н.А., Бурман Ю. М. Леммы Морса для функционалов вариационного исчисления // Функц. анализ и его прилож. 1971. Т. 25. № 3. С. 1-11.

[13] Попов Е. П. Нелинейные задачи статики тонких стержней. М.: ОГИЗ, 1948.

[14] Григолюк Э. И., Шалашилин В. И. Проблемы нелинейного деформирования: Метод продолжения по параметру в нелинейных задачах механики твердого деформируемого тела. М.: Наука, 1988.

[15] Заславский Г. М., Сагдеев Р. З. Введение в нелинейную физику. М.: Наука, 1988.

[16] Блехман И. И., Мышкис А. Д., Пановко Я. Г. Механика и прикладная математика: Логика и особенности приложений математики. М.: Наука, 1990.

[17] Пуанкаре А. Избранные труды в трех томах. Том 2: Новые методы небесной механики. Топология. Теория чисел. М.: Наука, 1972.

[18] Аппель П. Фигуры равновесия вращающейся однородной жидкости. М.-Л.: ОНТИ, 1936.

[19] Ляпунов A. M. Sur les figures d'équilibre peu differentes des éllipsöides d'une masse liquide homogène donée d'un mouvement de rotation, p. 1. С.-Петербург: Зап. Акад. наук, 1906.

[20] Schmidt E. Zur Theorie linearen und nichtlinearen Integralgleichungen, Theil 3, Über die Auflösung der nichtlinearen Integralgleichungen und Verzweigung ihrer Losungen // Math. Ann. 1908. P. 65.

[21] Вайнберг М.М., Треногин В.А. Теория ветвления решений нелинейных уравнений. М.: Наука.

[22] Conley C. C., Zehnder E. The Birkhoff-Lewis fixed point theorem and a conjecture of V.I. Arnol'd // Invent. Math. 1983. V. 73. P. 33-49.

[23] Болотин С. В. Периодические решения системы с гироскопическими силами // Прикл. матем. и механ. 1987. Т. 51. №4. С. 686-687.

[24] Борисович А. Ю. Редукция задачи о бифуркации минимальных поверхностей к операторным уравнениям и отыскание бифуркаций от катеноида, геликоида, поверхностей Шерка и Эннепера // УМН. 1986. Т. 41. № 5. С. 165-166.

[25] Койтер В.Т. Устойчивость и закритическое поведение упругих систем // Механика. Периодический сборник переводов иностр. статей 1960. № 5. С. 99-110. 
[26] Арнольд В. И., Варченко А.Н., Гусейн-Заде С. М. Особенности дифференцируемых отображений. Классификация критических точек каустик и волновых фронтов. М.: Наука, 1982.

[27] Арнольд В. И. Математические методы классической механики. М.: Наука, 1989.

[28] Годбийон К. Дифференциальная геометрия и аналитическая механики. М.: Мир, 1973.

[29] Рубановский В.Н., Самсонов В. А. Устойчивость стационарных движений в примерах и задачах. М.: Наука, 1988.

[30] Арнольд В.И., Козлов В. В., Нейштадт А. И. Математические аспекты классической и небесной механики // Итоги науки и техники. Сер. Современные проблемы математики. Фундаментальные направления. Т. 3. М.: ВИНИТИ, 1985. С. 1-304.

[31] Арнольд В.И. Гамильтоновость уравнений Эйлера динамики твердого тела и идеальной жидкости // УМН. 1969. Т. 24. № 3. С. 225-226.

[31.1] Мищенко А.С. Интегралы геодезических потоков на группах Ли // Функц. анализ и его прилож. 1970. Т. 4. №3. С. 73-78.

[31.2] Манаков С. В. Замечания об интегрируемости уравнений Эйлера динамики $n$-мерного твердого тела // Функц. анализ и его прилож. 1976. Т. 10. № 4. С. 93-94.

[31.3] Мищенко А. С., Фоменко А. Т. Уравнения Эйлера на конечномерных группах Ли // Изв. АН СССР. Сер. матем. 1978. Т. 42. № 2. С. 396-415.

[31.4] Фоменко А. Т. Дифференциальная геометрия и топология. Дополнительные главы. М.: Изд-во МГУ, 1983.

[31.5] Вишик С. В., Должанский $\Phi$. В. Аналоги уравнений Эйлера-Пуассона и магнитной гидродинамики, связанные с группами Ли // ДАН СССР. 1978. Т. 238. № 5. С. 1032-1035.

[31.6] Трофимов В. В., Фоменко А.Т. Интегрируемость по Лиувиллю гамильтоновых систем на алгебрах Ли // УМН. 1984. Т. 39. № 32. С. 3-56.

[31.7] Болсинов А. В. Критерий полноты семейства функций в инволюции, построенного методом сдвига аргумента // ДАН СССР. 1988. Т. 301. № 5. С. 1037-1040.

[31.8] Богоявленский О.И. Опрокидывающиеся солитоны. Нелинейные интегрируемые уравнения. М.: Наука, 1991.

[32] Сапронов Ю. И. Многомерные спящие волчки // Глобальный анализ и математическая физика. Воронеж: Изд-во ВГУ, 1987. С. 95-109.

[33] Сапронов Ю. И. Бифуркации стационарных вращений многомерного асимметричного твердого тела из режима спящего волчка // Глобальный анализ и нелинейные уравнения. Воронеж: Изд-во ВГУ, 1988. С. 141-152.

[34] Смейл С. Топология и механика // УМН. 1972. Т. 27. № 2. С. 77-133.

[35] Борисович Ю.Г., Звягин В.Г., Сапронов Ю.И. Нелинейные фредгольмовы отображения и теория Лере-Шаудера // УМН. 1977. Т. 32. № 4. С. 3-54.

[36] Banach S., Mazur S. Über mehrdeütige stetige Abbildungen // Studia Math. 1934. № 5. P. 174-178.

[37] Nashed M.Z., Hermander J. E. Global invertibility in nonlinear functional analysis // Fixed Point Theory and Applications. River Edge: World Scientific Publishing, NJ-1992. P. 229-247.

[38] Красносельский М.А., Вайникко Г. М., Забрейко П. П., Рутицкий Я.Б., Стеценко В.Я. Приближенные решения операторных уравнений. М.: Наука, 1969.

[39] Сапронов Ю.И. Нелокальные конечномерные редукции в вариационных краевых задачах // Матем. заметки. 1991. Т. 49. № 1. С. 94-103.

[40] Sapronov Yu. I. Smooth marginal analysis of bifurcation of extremals // Geometry in Partial Differential Equations. Singapore: World Scientific Publishing, 1994. P. 345-375.

[41] Клингенберг В. Лекция о замкнутых геодезических. М.: Мир, 1982.

[42] Weinstein A. Singularities of families of functions // Differential Geometrie in Grossen. 1971. V. 4. Oberwolfach. P. 323-330.

[43] Ляв А. Математическая теория упругости. М.-Л.: НКТН СССР, 1935. 
[44] Николаи Е. Л. К задаче об упругой линии двоякой кривизны // Труды по механике. М.: Гостехиздат, 1955. С. 45-277.

[45] Илюхин А. А. Пространственные задачи нелинейной теории упругих стержней. Киев: Наукова думка, 1979.

[46] Заваровский Ю.Н., Сапронов Ю.И. Нормальная форма ключевой функции в задачах о критических нагрузках упругих стержней. Воронеж, 1981. Деп. в ВИНИТИ. № 4185-81.

[47] Заваровский Ю.Н. Ветвление решений уравнения Кирхгофа симметричного пространственного стержня. Воронеж: ВГУ, 1981. Деп. в ВИНИТИ. № 4610-81.

[48] Заваровский Ю.Н. Нормальная форма ключевой функции обобщенного уравнения Кирхгофа // УМН. 1983. Т. 38. №3. С. 177-178.

[49] Левченко О.Н. Маргинальный анализ равновесия эйлерова стержня // Тезисы 6-й Межвузовской научной конференции молодых ученых. Липецк, 1992.

[50] Levchenko O. N., Sapronov Yu. I. Morse-Bott reduction for a symmetric Kirchhoff rood // Methods and Applications of Global Analysis. Voronezh Univ. Press, 1993. P. 95-100.

[51] Ворович И. И. Математические проблемы нелинейной теории пологих оболочек. М.: Наука, 1989.

[52] Вольмир А.С. Гибкие пластины и оболочки. М.: Гостехиздат, 1956.

[53] Волков Е. А. О решении краевых задач для уравнений Пуассона в прямоугольнике // ДАН СССР. 1962. Т. 147. № 2. С. 13-16.

[54] Holder E. J., Schaeffer D. Boundary conditions and mode jumping in the Karman equations // SIAM J. Math. Anal. 1984. V. 15. №3. P. 446-457.

[55] Сапронов Ю.И. Многомодовые бифуркации упругих равновесий // Прикл. матем. и механ. 1988. Т. 52. №6. С. 997-1006.

[56] Сапронов Ю.И. Двумодовая бифуркация решений уравнения Кармана // Дифференц. уравнения. 1989. Т. 25. №6. С. 1078-1081.

[57] Гнездилов А. В., Сапронов Ю. И. Нелокальные конечномерные редукции в теории изгиба тонких упругих пластин // Понтрягинские чтения - V. Тезисы докладов. Воронеж: Изд-во ВГУ, 1994. 